\title{
LA COHERENCIA EN EL DERECHO *
}

\author{
Amalia Amaya ** \\ Universidad Nacional Autónoma de México
}

RESUMEN. Este trabajo examina el concepto de coherencia y el papel que ésta juega en el razonamiento jurídico. En primer lugar, se ofrece un somero análisis de las teorías de la coherencia en el derecho, prestando atención a las teorías de la justificación tanto de los enunciados normativos como de los enunciados fácticos. En segundo lugar, se identifican una serie de problemas que afectan a las teorías de la coherencia en el derecho, tanto a las teorías que se ocupan de las cuestiones normativas como aquéllas que se centran en las cuestiones fácticas. En tercer lugar, se articula un modelo coherentista con el objetivo de solventar - 0 , al menos-mitigar los problemas que enfrentan las teorías coherentistas de la justificación jurídica. Según este modelo, una creencia acerca de los hechos o del derecho está justificada si y sólo si es óptimamente coherente, es decir, si es una creencia que un decisor jurídico epistémicamente responsable habría aceptado en virtud de su coherencia en circunstancias similares. Por último, se analizan las implicaciones del modelo de coherencia propuesto para una teoría general de la argumentación jurídica. cación.

Palabras clave: coherencia, responsabilidad epistémica, inferencia a la mejor expli-

ABSTRACT. This paper examines the concept of coherence and its role in legal reasoning. First, it gives an overview of the coherence theory in law, paying attention to coherence theories of the justification of both normative and factual propositions. Second, it identifies some problem-areas confronting coherence theories of legal reasoning about both disputed questions of fact and disputed questions of law. Third, with a view to solving these problems, it proposes a coherence model of legal reasoning. The main tenet of this model is that a belief about the law and the facts under dispute is justified if it is "optimally coherent», that is, if it is such that an epistemically responsible legal decision-maker would have accepted it as justified by virtue of its coherence in like circumstances. Last, this paper examines the implications of the version of legal coherentism proposed for a general theory of legal reasoning and rationality.

Keywords: coherence, epistemic responsibility, inference to the best explanation.

\footnotetext{
* Fecha de recepción: 6 de junio de 2011. Fecha de aceptación: 2 de julio de 2011.

** Muchas personas me han ayudado enormemente a desarrollar las ideas presentadas en este trabajo. Estoy especialmente agradecida a M. AtienZA, N. Chomsky, C. Elgin, F. Schauer, R. StAlnaker y M. La Torre.
} 


\section{INTRODUCCIÓN}

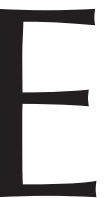

n las últimas décadas, se han propuesto diversas teorías coherentistas en distintos ámbitos. En epistemología, se han propuesto teorías coherentistas de la justificación como una alternativa al fundacionismo ${ }^{1}$. En algunas propuestas, no sólo la justificación epistémica sino también el concepto de verdad se han tratado de definir apelando a la noción de coherencia ${ }^{2}$. En el campo de la filosofía de la ciencia, la coherencia se ha considerado como un criterio fundamental para la selección de teorías científicas ${ }^{3}$. La coherencia ha figurado también de manera central en algunas teorías de la razón práctica así como en algunas de las principales teorías del razonamiento moral ${ }^{4}$. La coherencia ha jugado un papel importante incluso en algunas teorías en psicología y en lingüística, en donde se ha utilizado el concepto de coherencia para explicar fenómenos tan diversos como la formación de impresiones ${ }^{5}$, la interpretación del discurso ${ }^{6}$ y el pensamiento analógico ${ }^{7}$.

El coherentismo ha sido también una posición teórica muy influyente en la teoría del derecho contemporánea ${ }^{8}$. A pesar de que hay distintas opiniones acerca del lugar que debe ocupar la coherencia en una teoría de la justificación jurídica, la mayoría de los filósofos del derecho están de acuerdo en que la coherencia es, al menos, un ingrediente importante en la justificación jurídica. Sin duda, las teorías coherentistas de la justificación jurídica han contribuido de manera importante a la mejor comprensión del concepto de coherencia y del papel que ésta juega en la argumentación jurídica. Sin embargo, estas teorías presentan algunos problemas que es necesario atender. En primer lugar, la mayoría de las teorías de la coherencia en el derecho se han centrado en la función que cumple la coherencia en relación a la justificación de los enunciados normativos y no se han ocupado de manera sistemática de analizar el papel que des-

1 Vid., entre otros, L. BonJour, The Structure of Empirical Knowledge, Cambridge, Cambridge University Press, 1985; y K. LeHrer, Theory of Knowledge, 2. ${ }^{a}$ ed., Boulder, Wesview Press, 2000.

2 Para una revisión de la literatura acerca de las teorías de la verdad como coherencia, vid. R. C. S. WaLKER, The Coherence Theory of Truth, London, Routledge, 1989.

3 Vid., por ejemplo, P. Thagard, Conceptual Revolutions, Princeton, Princeton University Press, 1992.

4 Vid. H. RichardSOn, Practical Reasoning about Final Ends, Cambridge, Cambridge University Press, 1994; S. Hurley, Natural Reasons: Personality and Polity, Oxford, Oxford University Press, 1989; y P. Thagard y E. Millgram, «Deliberative Coherence», Synthese, 108, 1996. Acerca de las teorías coherentistas de la justificación moral, vid., J. RAwLS, A Theory of Justice, ed. rev. Cambridge, Harvard University Press, 1999; A. H. Goldman, Moral Knowledge, London, Routledge, 1988; y P. THAGard, «Ethical Coherence», Philosophical Psychology, 11, 1998.

5 Z. KundA, Social Cognition, Cambridge, MIT Press, 1999.

${ }^{6}$ Para una revisión de la literatura coherentista sobre la interpretación del discurso, vid., C. HeLLMAN, «The Notion of Coherence in Discourse», en G. RicKHeIT y C. HABEL (eds.), Focus and Coherence in Discourse Processing, Berlin, DeGruyter, 1995. 1995.

${ }^{7}$ K. J. Holyoak y P. Thagard, Mental Leaps: Analogy in Creative Thought, Cambridge, MIT Press,

8 Vid., entre otros, N. MACCormicK, «Coherence in Legal Justification», en W. KraWIETZ et al. (eds.), Theorie der Normen, Berlin, Duncker and Humblot, 1984; R. DworkIn, Law's Empire, London, Fontana, 1986; A. PeCzenik, On Law and Reason, Dordrecht, Kluwer, 1989; J. RAZ, «The Relevance of Coherence», Boston University Law Review, 72, 1992; L. WINTGENS, «On Coherence and Consistency», en W. KRAWIETZ et al. (eds.), The Reasonable as Rational? On Legal Argumentation and Justification, Berlin, Duncker and Humblot, 2000; A. AARNiO et al., On Coherence Theory in Law, Lund, Juristfölager i Lund, 1998; y J. C. HaGE, «Law and Coherence», Ratio Iuris, 17, 2004. 
empeña la coherencia en la justificación de los enunciados fácticos. En segundo lugar, las teorías de la coherencia en el derecho han adoptado fundamentalmente un punto de vista normativo y han prestado poca atención a los estudios de psicología cognitiva acerca de cómo razonan los decisores jurídicos. Por último, las teorías de la coherencia en el derecho son vulnerables a una serie de objeciones clásicas que se han dirigido en contra de las teorías coherentistas de la justificación.

En este trabajo — que es una versión reducida de un trabajo mucho más amplio— ${ }^{9}$ quisiera explorar la posibilidad de desarrollar una teoría de la coherencia para el derecho que sea potencialmente aplicable al razonamiento tanto acerca de normas como de hechos; que tome en cuenta algunos de los estudios de carácter empírico que se han realizado recientemente sobre el razonamiento jurídico; y que tenga la capacidad de resolver (o, al menos, de mitigar) las objeciones que se pueden dirigir en contra de una concepción coherentista de la justificación jurídica. En resumen, según el modelo de coherencia que quisiera presentar aquí, una creencia acerca de los hechos o el derecho está justificada si es «óptimamente coherente», es decir, si es una creencia que un decisor jurídico epistémicamente responsable podría haber aceptado como justificada en circunstancias similares. Un decisor jurídico es epistémicamente responsable en la medida en que cumpla con una serie de deberes epistémicos y lleve a cabo las actividades de investigación y deliberación propias de su cargo de una manera epistémicamente virtuosa. Según este modelo, los decisores jurídicos forman creencias acerca de los hechos y del derecho que están justificadas en virtud de su coherencia por medio de una inferencia explicativa. En esta propuesta, la coherencia se considera una cuestión de satisfacción de restricciones positivas y negativas y un estándar de justificación que depende de factores contextuales. De acuerdo con este modelo, la justificación jurídica es, por tanto, una cuestión de coherencia óptima.

El argumento de este trabajo se desarrollará de la siguiente manera. En las secciones 2 y 3 presentaré de manera somera las teorías coherentistas de la justificación de los enunciados normativos y fácticos en el derecho, respectivamente. En la sección 4 describiré algunos de los problemas que presentan las teorías de la coherencia en el derecho, tanto acerca de normas como de hechos. En la sección 5, presentaré los elementos principales de la propuesta coherentista que quisiera defender en este trabajo. En las secciones 6 y 7 , examinaré los problemas del coherentismo a la luz del modelo de coherencia articulado anteriormente y presentaré una propuesta de solución. En la sección 8 se discutirán algunas de las implicaciones del modelo de coherencia propuesto para la teoría de la argumentación jurídica y, en general, para la teoría del derecho. Por último, en la sección 9, apuntaré algunos de los problemas que presenta la concepción coherentista de la justificación jurídica defendida en este trabajo.

\section{COHERENCIA, JUSTIFICACIÓN E INTERPRETACIÓN JURÍDICA}

Las teorías de la coherencia han ocupado un lugar central en la filosofía del derecho contemporánea. En lo que sigue, presentaré de manera somera el estado de la

9 Vid. A. AmaYA, The Tapestry of Reason: An Inquiry into the Nature of Coherence and its Role in Legal Argument, Oxford, Hart Publishing, en prensa. 
cuestión en la teoría de la coherencia en el derecho. Comenzaré discutiendo brevemente las teorías de la coherencia normativa, es decir, las teorías coherentistas de la justificación de los enunciados normativos en el derecho.

Hay una gran variedad de teorías coherentistas de la justificación jurídica. Mientras que algunos teóricos del derecho definen la justificación jurídica exclusivamente en términos de coherencia, otros le dan a la coherencia un papel más modesto y consideran que la coherencia es un ingrediente fundamental, aunque no el único, de la justificación jurídica. Es posible, por tanto, distinguir entre teorías «fuertes» de la coherencia, según las cuales la coherencia es una condición necesaria y suficiente de la justificación jurídica y teorías «débiles» de la coherencia, que sostienen que la coherencia es una condición necesaria pero no suficiente de la justificación jurídica ${ }^{10}$. Entre las distintas teorías que analizan la noción de justificación jurídica apelando a la idea de coherencia, me centraré en tres propuestas particularmente influyentes: la teoría de la coherencia normativa de N. MACCORMICK ${ }^{11}$, el modelo de la ponderación de A. PECZENIK $^{12}$ y la teoría del derecho como integridad de R. DwORKIN ${ }^{13}$. Como estas teorías son bien conocidas, presentaré tan sólo los rasgos centrales de las mismas ${ }^{14}$.

Una discusión de las teorías de la coherencia en el derecho no puede sino empezar con el trabajo de N. MACCORMICK, pionero en los estudios acerca de la coherencia y la justificación jurídica. MACCORMICK es uno de los pocos teóricos del derecho que han examinado el papel que juega la coherencia tanto en relación a la justificación de los enunciados fácticos como normativos en el derecho. MACCORMICK diferencia entre la «coherencia normativa», es decir, el tipo de coherencia que es relevante para la justificación de conclusiones acerca de cuestiones de derecho y la «coherencia narrativa», que es el tipo de coherencia que es relevante para la justificación de conclusiones acerca de cuestiones de hecho. Según MACCORMICK, la coherencia normativa —me ocuparé de la coherencia narrativa más adelante- es la propiedad de un conjunto de normas que pueden ser explicadas en base a una serie de principios generales que delinean una forma de vida satisfactoria. La coherencia normativa juega un papel importante pero limitado en la justificación de las decisiones judiciales en los casos difíciles. Según MACCORMicK, los argumentos de coherencia permiten identificar un conjunto de deci-

10 En filosofía, la «teoría de la justificación como coherencia» se refiere exclusivamente a las teorías «fuertes» de la coherencia.

11 Vid. N. MACCORMICK, «Coherence in Legal Justification», op. cit.; N. MaCCormicK, «Argumentation and Interpretation in Law», Ratio Iuris, 6, 1993; N. MACCORMICK, Legal Reasoning and Legal Theory, Oxford, Clarendon Press, 1994; N. MacCormick, Rhetoric and the Rule of Law: A Theory of Legal Reasoning, Oxford, Oxford University Press, 2005.

12 A. PECZENIK, «A Coherence Theory of Juristic Knowledge», On Coherence Theory of Law, op. cit.; A. PECZENIK, «Certainty or Coherence?», The Reasonable as Rational? op. cit.; A. PECZENIK, «Coherence, Truth, and Rightness in the Law», en P. NERHOT (ed.), Law, Interpretation, and Reality, Dordrecht, Kluwer, 1990; A. PECZENIK, «Law, Morality, Coherence, and Truth», Ratio Iuris, 7, 1994; «The Passion for Reason: Some Remarks on Coherence in Law», en E. GARZÓN et al. (eds.), Normative Systems in Legal and Moral Theory, Berlin, Ducker and Humblot, 1997; A. PECZENIK, «The Passion for Reason», en L. J. WINTGENS (ed.), The Law in Philosophical Perspectives, Dordrecht, Kluwer, 1999.

13 R. Dworkin, A Matter of Principle, Cambridge, Harvard University Press, 1985; DworkIN, Law's Empire, op. cit.; R. DwOrkin, «No Right Answer?», en P. M. S. Hacker y J. RAz (eds.), Law, Morality and Society, Oxford, Clarendon Press, 1972; R. DwORKIN, «Objectivity and Truth: You'd Better Believe it», Philosophy and Public Affairs, 25, 1996, y R. DwORKIN, «Thirty Years On», Harvard Law Review, 115, 2002.

14 Para una discusión detallada de estas teorías, vid. AmaYA, The Tapestry of Reason, op. cit., capítulo 1. 
siones como «justificables» pero la justificación de las mismas depende, en último término, de argumentos consecuencialistas. En este sentido, la teoría de MACCORMICK es una teoría de la coherencia «débil» ya que postula que la coherencia es una condición necesaria, pero no suficiente, de la justificación jurídica. En todo caso, a pesar de que la coherencia no nos permita justificar plenamente una decisión, según MACCORMICK, la coherencia es un valor esencial en el derecho en cuanto que contribuye de manera importante a realizar los ideales de justicia formal.

PECZENIK ha defendido una teoría de la argumentación jurídica que otorga a la coherencia un papel más amplio en la justificación. PECZENIK — junto con ALEXYha formulado un conjunto de criterios para evaluar el grado de coherencia de una teoría. En concreto, según ALEXY y PECZENIK, la coherencia de una teoría depende de criterios que determinan las propiedades de la estructura de soporte de la misma, de criterios relativos a los conceptos empleados por la teoría así como de criterios relacionados con las propiedades del ámbito de aplicación de la teoría ${ }^{15}$. PECZENIK sostiene que la coherencia se construye a lo largo del proceso de decisión judicial por medio de una operación de ponderación. La justificación jurídica es el producto del balance coherente de un conjunto de razones relevantes, que incluye tanto razones morales como razones jurídicas. No obstante, la forma en que PECZENIK concibe la operación de ponderación limita de manera significativa la capacidad de la coherencia de generar justificación. Para PECZENIK, el último paso en la operación de ponderación se basa, en último término, en las preferencias individuales y personales. La coherencia con un conjunto de razones relevantes hace que las decisiones sean no simplemente justificables — como sostendría MACCORMicK - sino plenamente justificadas. Sin embargo, en los casos en los que el balance coherente de razones permite justificar más de una decisión, los estándares coherentistas de la justificación no pueden guiar a los decisores jurídicos acerca de qué decisión deben aceptar como justificada. Desde el punto de vista de PECZENIK, las razones últimas que apoyan una decisión no son sino una cuestión que depende de las preferencias subjetivas de los decisores jurídicos.

Por último, la teoría del derecho como integridad ha sido ampliamente interpretada como una aproximación coherentista al derecho. Esta teoría está profundamente marcada por los compromisos coherentistas de DwORKIN. A mi modo de ver, DwORKIN sostiene una teoría coherentista de la justificación según la cual una decisión jurídica está justificada si es coherente con el conjunto de principios que mejor explica y justifica la práctica jurídica, a la luz de una teoría de la moralidad política. DwORKIN define no sólo la justificación jurídica, también —-me parece- el concepto de verdad en el derecho en términos coherentistas. La teoría interpretativa del derecho de DwORKIN está comprometida con una teoría de la verdad como coherencia según la cual una proposición jurídica particular es verdadera si pertenece a la teoría que explica y justifica el derecho de manera más coherente. Por tanto, según DwORKIN, tanto la justificación de las proposiciones jurídicas como la verdad de las mismas dependen de su coherencia con un conjunto de creencias interpretativas acerca del derecho y de la moralidad política. Las razones de coherencia, desde este punto de vista, nos permiten identificar (en la mayor parte de los casos) una única respuesta como justificada así

15 Vid. R. AlEXY y A. PeCZENIK, «The Concept of Coherence and its Significance for Discourse Rationality», Ratio Iuris, 3, 1990. 
como determinar de manera completa las condiciones de verdad de las proposiciones normativas en el derecho.

La motivación principal de las teorías coherentistas de la justificación de juicios normativos, me parece, es expandir el ámbito de la razón en el derecho más allá de los límites impuestos por las aproximaciones formalistas al razonamiento jurídico. Estas teorías tratan de avanzar una concepción más amplia de la razón en el derecho, exigiendo que las decisiones jurídicas satisfagan los criterios de coherencia en casos en los que, según las versiones formalistas del positivismo jurídico, los decisores jurídicos gozan de discreción. Las propuestas coherentistas discutidas anteriormente amplían el ámbito de aplicación de la razón en el derecho en distintas medidas. Según MACCoRMICK, las decisiones jurídicas deben ser coherentes, además de ser consistentes con el derecho vigente. Esto restringe de manera importante la discreción de los decisores jurídicos, en cuanto que los argumentos de coherencia reducen el conjunto de decisiones justificables. Sin embargo, en los casos en los que varias respuestas son igualmente coherentes con el derecho aplicable, los decisores jurídicos han de decidir en base a argumentos consecuencialistas, los cuales tienen — según MACCORMICK - una naturaleza subjetiva. Por tanto, el alcance de la propuesta de MACCORMICK en relación con el objetivo de expandir el espacio de la razón en el derecho es relativamente modesto. La teoría de PECZENIK nos permite avanzar un paso más en la dirección de constreñir a los decisores jurídicos. Según MACCORMicK, las evaluaciones subjetivas entran en juego en cuanto hay dos decisiones que son igualmente coherentes con el derecho. A diferencia de MACCORMick, en la propuesta de PECZENIK, las consideraciones de tipo evaluativo sólo juegan un papel en situaciones en las que hay dos decisiones que son igualmente coherentes con el conjunto de razones tanto jurídicas como morales. Por tanto, PECZENIK requiere que los decisores jurídicos satisfagan unas condiciones de coherencia más exigentes que las impuestas por MACCORMicK. Sin embargo, al considerar que el último paso en la deliberación depende de preferencias subjetivas, PECZENIK limita de manera importante la ambición coherentista de expandir el ámbito de la razón en el derecho. Por último, DwORKIN lleva a los extremos el proyecto coherentista de aumentar el dominio de la razón en el derecho al defender la tesis de que, salvo en casos excepcionales, las razones de coherencia nos permiten identificar una única decisión como justificada.

Mientras que MACCoRmick y PeCZENIK parecen estar demasiado conscientes de los límites de la razón en el derecho, DwORKIN, por el contrario, parece ser excesivamente optimista acerca de la capacidad de la razón para guiar y constreñir los procesos de toma de decisiones jurídicas. A la luz del último objetivo del coherentismo, a saber, proporcionar una alternativa no escéptica a las concepciones formales del razonamiento jurídico, lo que sería deseable es contar con una concepción coherentista del derecho que le otorgue una función amplia a la razón en el derecho pero sin subestimar la seriedad de las objeciones escépticas. Por tanto, a pesar de que estas tres teorías han impulsado de manera fundamental el proyecto de ofrecer una visión no reduccionista del ámbito de la razón en el derecho, las aspiraciones coherentistas no han sido todavía plenamente satisfechas. Además, aunque estas teorías han contribuido significativamente a una mejor comprensión del concepto de coherencia y del papel que ésta juega en el razonamiento jurídico, no han logrado solventar algunos de los problemas tradicionales que afectan a las teorías de la coherencia. Me ocuparé de analizar estos 
problemas en detalle más adelante, pero antes examinemos brevemente algunas teorías de la prueba según las cuales la coherencia juega un papel central en la justificación de los enunciados fácticos en el derecho.

\section{COHERENCIA Y PRUEBA EN EL DERECHO}

En el contexto del razonamiento acerca de los hechos, no hay una teoría de la coherencia que tenga un grado de desarrollo similar al de las teorías de la coherencia normativa. Como se indicó anteriormente, mientras que las cuestiones relativas al papel que juega la coherencia normativa en la justificación jurídica han sido ampliamente discutidas en la literatura, la función de la coherencia en la justificación de conclusiones acerca de los hechos en el derecho ha recibido una atención mucho menor. No obstante, la coherencia figura de manera prominente en algunas teorías de la prueba.

Las teorías coherentistas de la prueba pretenden ser una alternativa a las teorías probabilistas de la prueba, en concreto, al bayesianismo ${ }^{16}$. El modelo bayesiano de la prueba jurídica está basado en una concepción de la racionalidad de los decisores jurídicos según la cual ser racional consiste en razonar conforme a los axiomas de la teoría de la probabilidad, una de cuyas consecuencias lógicas fundamentales es el teorema de Bayes. En este sentido, las teorías coherentistas de la justificación de los enunciados fácticos en el derecho, al igual que las teorías de la coherencia normativa, tratan de superar una concepción formalista de la racionalidad jurídica. Estas teorías se han propuesto en tres ámbitos diferentes: la teoría de la prueba, la teoría del derecho y la psicología del razonamiento jurídico.

En el ámbito de la teoría de la prueba, hay una serie de juristas que han defendido teorías holistas de la evaluación de las pruebas ${ }^{17}$. Según el holismo, la fuerza probatoria de las pruebas disponibles debe ser valoradas en su conjunto ${ }^{18}$. Desde este punto de vista, no es posible aceptar un enunciado probatorio como justificado de manera aislada, sino que es el conjunto de pruebas, hipótesis y creencias generales el que confiere

16 Vid. P. Tillers y E. D. GreEn (eds.), Probability and Inference in the Law of Evidence: The Uses and Limits of Bayesianism, Dordrecht, Kluwer, 1988. Dos conferencias que se ocuparon de manera exhaustiva de la teoría bayesiana de la prueba son: «Decision and Inference in Litigation», Cardozo Law Review, 13, 1991, y «Bayesianism and Juridical Proof», International Journal of Evidence and Proof, 1, 1997. Algunas monografías importantes son las siguientes: D. A. SCHUM, The Evidential Foundations of Probabilistic Reasoning, Evanston, Northwestern University Press, 2001; J. B. KadANE y D. A. Schum, A Probabilistic Analysis of the Sacco and Vanzetti Evidence, New York, Wiley and Sons, 1996; B. RoberTson y G. A. Vignaux, Interpreting Evidence, Chichester, Wiley and Sons, 1995; y J. L. GASTwIRTH (ed.), Statistical Science in the Courtroom, New York, Springer-Verlag, 2000.

${ }_{17}$ Vid. R. Allen, «The Nature of Juridical Proof», Cardozo Law Review, 13, 1991; R. Allen, «Factual Ambiguity and a Theory of Evidence», Northwestern University Law Review, 58, 1994; R. AlLEN, «Rationality, Algorithms and Juridical Proof», International Journal of Evidence and Proof, 1, 1997; M. S. PARDO, «Juridical Proof, Evidence and Pragmatic Meaning: Toward Evidentiary Holism», Northwestern University Law Review, 95, 2000; P. TILleRS, «Mapping Inferential Domains», Boston University Law Review, 66, 1986; y P. TIllers, «Webs of Things in the Mind: A New Science of Evidence», Michigan Law Review, 87, 1989. Para una breve pero iluminadora exposición crítica de las teorías holistas, vid. W. TwINING, Theories of Evidence Bentham and Wigmore, Stanford, Stanford University Press, 1985, 183-185; W. TwINING, Rethinking Evidence: Exploratory Essays, Cambridge, Cambridge University Press, 2006 (2. ${ }^{\text {e ed.) }), 306-311 . ~}$

18 R. DAMAŠKA, «Atomistic and Holistic Evaluation of Evidence: A Comparative View», en D. S. Clark (ed.), Comparative and Private International Law, Berlin, Duncker and Humblot, 1990, 91. 
justificación a cada uno de estos enunciados. Por tanto, el holismo está implícitamente fundamentado en una concepción coherentista de la justificación según la cual una creencia está justificada si pertenece a un sistema coherente de creencias.

A pesar de que en el siglo XVIII hubo algunos precursores del holismo, solo recientemente éste ha sido una posición teórica importante en el derecho de la prueba ${ }^{19}$. En la teoría de la prueba contemporánea, la teoría holista más influyente es, probablemente, la teoría de la «plausibilidad relativa» propuesta por R. ALLEN ${ }^{20}$. Según esta teoría «el razonamiento acerca de los hechos en el derecho consiste en la determinación de la plausibilidad comparada de las explicaciones ofrecidas por las partes en el juicio [...]. En los casos civiles, el juzgador de los hechos debe identificar la explicación más plausible de los eventos relevantes, mientras que en los casos penales, el fiscal debe proporcionar una explicación plausible de la culpabilidad y demostrar que no existe una explicación plausible de la inocencia» ${ }^{21}$. Para ALLEN, la plausibilidad de una historia depende de variables tales como su coherencia, grado de completud, singularidad, economía y probabilidad ${ }^{22}$. Esta aproximación es holista ya que las teorías propuestas por las partes deben ser aceptadas o rechazadas en su conjunto, teniendo en cuenta el estándar de prueba aplicable ${ }^{23}$.

Otra aproximación holista a la evaluación de las pruebas que merece la pena destacar es la teoría pragmática propuesta por M. PARDO. Esta teoría se basa en la tesis holista de QuiNE acerca del significado, según la cual el significado de cualquier enunciado es una función del papel que éste juega en una teoría del lenguaje considerada en su conjunto ${ }^{24}$. PARDO sostiene que el proceso probatorio requiere la interpretación pragmática de un conjunto de enunciados acerca de las pruebas disponibles. Durante un juicio, las partes proponen interpretaciones pragmáticas alternativas de varios enunciados probatorios. Después, el juez o jurado organiza estos enunciados en la forma de narrativas o esquemas probatorios y elige la narrativa que explica mejor los mismos. Según PARDO, dicha elección se hace en base a «principios básicos de racionalidad, tales como cobertura, coherencia y singularidad» ${ }^{25}$. A la luz de esta narrativa, el juez o jurado asigna significado pragmático a los distintos enunciados probatorios ${ }^{26}$. Sólo entonces es posible determinar el valor probativo de las pruebas, en función del papel que las mismas juegan en el esquema probatorio desarrollado previamente.

La aproximación holista a la evaluación de las pruebas (y la epistemología coherentista en la que ésta se basa) goza de un alto grado de plausibilidad psicológica ${ }^{27}$.

19 M. ABU-HAREIRA, «An Early Holistic Conception of Judicial Fact-finding», Juridical Review, 79, 1986.

20 Vid. Allen, «The Nature of Juridical Proof», op. cit.; Allen, «Factual Ambiguity and a Theory of Evidence», op. cit.

${ }_{21}$ R. J. Allen y B. LeiteR, «Naturalized Epistemology and the Law of Evidence», Virginia University Law Review, 87, 2001, 1528.

${ }_{22}$ R. Allen, «Rationality, Algorithms, and Juridical Proof: A Preliminary Inquiry», op. cit., 273.

23 AlLEN, «Factual Ambiguity and a Theory of Evidence», op. cit., 609.

${ }^{24}$ Para una discusión de las principales teorías del holismo acerca del significado, vid. J. Fodor y E. LePORE, Holism: A Shopper's Guide, Oxford, Blackwell, 1992.

25 PARDO, op. cit., 435.

${ }^{26} \mathrm{El}$ «significado pragmático», según PARDO, es «el significado extra-semántico que el hablante añade a las oraciones (o como el hablante usa las oraciones)», id., 429.

27 Vid. R. Hastie y N. Pennington, «A Cognitive Theory of Juror Decision-Making: The Story Model», Cardozo Law Review, 13, 1991; W. A. WagenaAR, P. J. van Koppen y H. F. M. Crombag, Anchored Narra- 
Una serie importante de estudios empíricos demuestran que los jueces y jurados razonan acerca de las pruebas a partir de un conjunto de hipótesis que tienen forma narrativa. Por tanto, estos estudios establecen que la «coherencia narrativa» juega un papel importante en el razonamiento jurídico acerca de los hechos. Dos propuestas me parecen particularmente significativas: el modelo de la historia, desarrollado por HASTIE y PENNINGTON, y la teoría de las «narrativas ancladas», elaborada por WAGENAAR, KOPPEN y CROMBAG ${ }^{28}$.

La tesis principal del modelo de la historia es que un proceso cognitivo central en la toma de decisiones por parte de jurados es la construcción de «estructuras narrativas» para organizar e interpretar las pruebas ${ }^{29}$. Según el modelo de la historia, los jurados aceptan la historia que, entre una serie de historias alternativas, mejor explica el conjunto de las pruebas. HASTIE y PENNINGTON consideran que hay tres «principios de certeza» que gobiernan la selección de una historia como la mejor, a saber, «cobertura», es decir, el grado en el cual una historia tiene la capacidad de dar cuenta de las pruebas presentadas en el juicio; «coherencia», que es un criterio que tiene a su vez tres componentes, consistencia, plausibilidad y completud; $\mathrm{y}$ «singularidad», ya que el grado de creencia en una historia se reduce si hay múltiples historias coherentes ${ }^{30}$. Por tanto, según el modelo de la historia, los jurados usan la coherencia narrativa como un criterio central para evaluar la aceptabilidad de la historia de la que va a depender su decisión.

La teoría de las narrativas ancladas examina el papel que juegan las narrativas en el razonamiento judicial en casos penales ${ }^{31}$. La tesis central de la teoría de las narrativas ancladas es que los jueces toman sus decisiones en casos penales en base a dos juicios:

tives: The Psychology of Criminal Evidence, New York, St. Martin's Press, 1993; y D. SIMON, «A Third View of the Black Box: Cognitive Coherence in Legal Decision-Making», The University of Chicago Law Review, 71, 2004.

${ }^{28}$ Tanto el modelo de la historia como la teoría de las narrativas ancladas le deben mucho al trabajo pionero de BENNETT y FeLDMAN en el ámbito de la psicología social. Vid. W. L. BenNeTt y M. S. Feldman, Reconstructing Reality in the Courtroom: Justice and Judgment in American Culture, New Brunswick, Rutgers University Press, 1981. El trabajo de BenNeTt y FeLDMAn también ha influido de manera importante en algunas aproximaciones coherentistas al razonamiento jurídico acerca de los hechos desarrollado por algunos teóricos del derecho, como B. JACKSON. Vid. infra en esta sección.

29 Vid. R. Hastie y N. Pennington, «Evidence Evaluation in Complex Decision-Making», Journal of Personality and Social Psychology, 51, 1986; R. HAstie y N. Pennington, «A Cognitive Theory of Juror Decision-Making: the Story Model», Cardozo Law Review, 1991; R. Hastie y N. Pennington, «Explaining the Evidence: Tests of the Story Model for Juror Decision-Making», Journal of Personality and Social Psychology, 1992; R. Hastie y N. Pennington, «Reasoning in Explanation-Based Decision-Making», Cognition, 49, 1993; R. Hastie y N. Pennington, «Explanation-Based Decision-Making», en T. Connoliy et al. (ed.), Judgment and Decision-Making: An Interdisciplinary Reader, 2. ${ }^{a}$ ed., Cambridge, Cambridge University Press, 2000; R. Hastie y N. Pennington, «The Story Model for Juror Decision-Making», en por R. HAstie (ed.), Inside the Juror: The Psychology of Juror Decision-Making, Cambridge, Cambridge University Press, 1993.

30 Vid. S. A. SLOMAN, «When Explanations Compete: the Role of Explanatory Coherence on Judgments of Likelihood», Cognition, 52, 1994.

31 WagenaAR, van Koppen y CROMbag, op. cit. Para una breve descripción de las tesis principales de esta teoría, vid. W. A. WAGENAAR, «Anchored Narratives: A Theory of Judicial Reasoning, and its Consequences», en G. Davies, S. Lloyd-Bostock, M. McMurran y C. Wilson (eds.), Psychology, Law, and Criminal Justice, Berlin, Walter de Gruyter, 1996. Para una crítica de la teoría de las narrativas ancladas, vid. W. TwinING, «Anchored Narratives: A Comment», The Great Juristic Bazaar, Darmouth, Ashgate, 2002; B. JACKSON, «"Anchored Narratives" and the Interface of Law, Psychology and Semiotics», Review of Anchored Narratives, por W. A. Wagenaar et al., Legal and Criminological Psychology, 1, 1996; M. Den Boer, Review of Anchored Narratives, por W. A. WAGENAAR et al., International Journal for the Semiotics of Law, 8, 1995; J. R. SPENCER, 
la evaluación de la plausibilidad de la historia presentada por el fiscal y la evaluación acerca de si esta narrativa está anclada de manera adecuada en creencias de sentido común que son generalmente aceptadas como verdaderas ${ }^{32}$. Mientras que el primer juicio evaluativo determina la «bondad» de la historia, el segundo determina el grado en el que la misma está apoyada o anclada en los hechos. Estos dos juicios, de manera conjunta, dan lugar a una evaluación de la credibilidad de la historia del fiscal, que es de lo que depende — según estos autores— la decisión final del juez ${ }^{33}$.

Tanto el modelo de la historia como la teoría de las narrativas ancladas son modelos psicológicos del proceso de toma de decisiones jurídicas que giran alrededor de la noción de historia. En ambos casos, la toma de decisiones acerca de los hechos se entiende como un proceso en el que se elaboran historias que nos permitan explicar las pruebas aducidas y se comparan las mismas en base, entre otros criterios, a su grado de coherencia. Un conjunto de estudios empíricos llevado a cabo recientemente por K. HolyoaK, D. Simon y S. ReAd ha planteado una visión diferente del tipo de coherencia que es relevante para el razonamiento acerca de los hechos en el derecho ${ }^{34}$. Según esta propuesta, denominada «razonamiento basado en la coherencia», el concepto de coherencia que es central en una teoría descriptiva del razonamiento jurídico acerca de los hechos no es el de coherencia narrativa sino la noción de coherencia como satisfacción de restricciones.

La teoría del «razonamiento basado en la coherencia» postula que la toma de decisiones jurídicas es un proceso mediante el cual los decisores reconstruyen la representación mental del problema a resolver y convierten un problema de decisión difícil en uno fácil, permitiendo así que las decisiones se tomen con un alto grado de confianza ${ }^{35}$.

«Anchored Narratives», Reseña de Anchored Narratives, por W. A. WAGENAAR et al., Cambridge Law Journal, 53, 1994.

32 Algunos ejemplos de anclas serían las siguientes: «los testigos dicen la verdad la mayor parte de las veces», «si un juguete hace ruido es porque alguien lo ha pisado», «los patólogos casi nunca se equivocan», «en general, la gente nunca entra en casa ajena sin el conocimiento y el consentimiento de su dueño con buenas intenciones».

33 WAGENAAR et al., op. cit., 10. Es importante destacar que la tesis principal de la teoría de las narrativas ancladas se diferencia de manera importante de la tesis central del modelo de la historia. Mientras que el modelo de la historia (y otras teorías como la de ALLEN) sostienen que los decisores jurídicos evalúan la plausibilidad de las historias del fiscal y de la defensa, «la teoría de las narrativas ancladas no asume que los jueces consideren dos narrativas, la del fiscal y la de la defensa, las cuales tendrían que considerarse igualmente relevantes hasta el final de la decisión, sino que, por el contrario, la teoría predice que es sobre todo la versión del fiscal acerca de lo que ocurrió la que será considerada y verificada en base a las pruebas presentadas por las dos partes», $i d$., 210.

34 Vid. K. J. Holyoak y D. Simon, «Bidirectional Reasoning in Decision-Making by Constraint Satisfaction», Journal of Experimental Psychology: General, 128, 1999; K. J. HOLYOAK et al., «The Emergence of Coherence Over the Course of Decision-Making», Journal of Experimental Psychology: Learning, Memory, and Cognition, 27, 2001; D. SimON y K. J. HolYOAK, «Structural Dynamics of Cognition: From Consistency Theories to Constraint Satisfaction», Personality and Social Psychology Review, 6, 2002; S. J. READ, C. J. SNOW y D. SimON, «Constraint Satisfaction Processes in Social Reasoning», en R. Alterman y D. KIRCH (eds.), Proceedings of the 25th Annual Conference of the Cognitive Science Society, Mahwah, Earlbaum, 2003; D. SimON, C. J. SNOw y S. J. READ, «The Redux of Cognitive Consistency Theories: Evidence Judgments by Constraint Satisfaction», Journal of Personality and Social Psychology, 86, 2004. Para un resumen de este conjunto de estudios empíricos, vid. D. SIMON, «A Third View of the Black Box: Cognitive Coherence in Legal Decision-Making», University of Chicago Law Review, 71, 2004.

35 Esta teoría es un modelo de toma de decisiones complejas. Las decisiones complejas son aquéllas en las que «las consideraciones constitutivas son muchas, contradictorias, ambiguas e inconmesurables». Vid. SIMON, «A Third View of the Black Box», op. cit., 516. La mayoría de los casos jurídicos que acaban en los tribunales 
De manera más precisa, SimON, HOLYOAK y sus colaboradores sostienen que en el proceso de toma de decisiones, la representación mental de la tarea del decisor jurídico sufre un proceso de cambio gradual hasta que, al final, alcanza un estado de coherencia con una de las alternativas de decisión, es decir, un estado en el que las consideraciones $\mathrm{o}$ «restricciones» que apoyan la alternativa elegida son firmemente aceptadas mientras que la fuerza de aquéllas que apoyan la alternativa rechazada es disminuida. A este punto, la decisión se sigue de la representación coherente con facilidad y confianza.

Los estudios que acabo de describir brevemente —el modelo de la historia, la teoría de las narrativas ancladas y la teoría del razonamiento basado en coherenciale dan a la teoría holista de la prueba un apoyo empírico bastante sólido. Todos estos estudios muestran que la coherencia - la coherencia narrativa y, más recientemente, la coherencia como satisfacción de restricciones- es un criterio fundamental para evaluar la plausibilidad de las representaciones que determinan las decisiones de los jueces y jurados acerca de cuestiones de hecho. Sin embargo, los resultados experimentales también revelan que razonar acerca de los hechos mediante la construcción y comparación de la coherencia relativa de representaciones alternativas de los mismos entraña riesgos importantes. Por tanto, para que la teoría de la coherencia sea una alternativa plausible al probabilismo, es necesario mostrar que ésta no es sólo una teoría psicológicamente plausible sino también atractiva desde un punto de vista normativo. Algunos teóricos del derecho han defendido justamente la tesis de que la coherencia, en su variedad narrativa, es un criterio importante de la justificación de las conclusiones acerca de cuestiones fácticas en el derecho ${ }^{36}$.

Hay varias teorías que explican la justificación jurídica en términos de coherencia narrativa. Una aproximación especialmente interesante es la propuesta por N. MACCORMiCK $^{37}$. Según MACCORMicK, la coherencia narrativa es la propiedad de un conjunto de proposiciones fácticas que «tiene sentido» en su totalidad. De manera más precisa, lo que hace que una historia sea coherente — dice MACCORMicK- es el hecho de que sea explicable por un conjunto de principios explicativos de tipo causal y motivacional ${ }^{38}$. Para MACCORMICK, la coherencia narrativa juega una función muy importante en la justificación de los enunciados fácticos en el derecho ${ }^{39}$. En concreto,

de apelación son complejos en este sentido. Este modelo ha sido aplicado a los procesos de decisión judicial en las instancias de apelación así como a los juicios por jurado. Aquí, me centro exclusivamente en las aplicaciones de esta teoría al razonamiento acerca de los hechos. Para las aplicaciones de la misma al razonamiento judicial en casos de apelación, vid. D. SimON, «A Psychological Theory of Legal Decision-Making», Rutgers Law Journal, 30, 1998; D. SIMON, «Freedom and Constraint in Adjudication: A Look Though the Lens of Cognitive Psychology», Brooklyn Law Review, 67, 2002.

36 Vid. MACCORMICK, «Coherence in Legal Justification», op. cit.; N. MACCORMICK, «The Coherence of a Case and the Reasonableness of Doubt», Liverpool Law Review, 2, 1980; B. JACKSON, Law, Fact and Narrative Coherence, Liverpool, Deborah Charles Publications, 1988; B. JACKSON, Making Sense in Law: Linguistic, Psychological, and Semiotic Perspectives, Liverpool, Deborah and Charles Publications, 1996; y B. van RoERMUnD, Law, Narrative and Reality, Dordrecht, Kluwer, 1997.

37 Acerca de la teoría de la coherencia narrativa de MACCormick, vid. MACCORMicK, Legal Reasoning and Legal Theory, op. cit., 89-92; MACCORMICK, «The Coherence of a Case and the Reasonableness of Doubt», op. cit.; MACCORMICK, «Coherence in Legal Justification», op. cit.; N. MACCORMICK, Rhetoric and the Rule of Law: A Theory of Legal Reasoning, Oxford, Oxford University Press, 2005, capítulo 11.

38 MACCORMICK, «Coherence in Legal Justification», op. cit., 50.

39 Para una crítica de la tesis de MACCORMICK según la cual la coherencia juega un papel importante en la justificación de conclusiones acerca de los hechos en el derecho, vid. L. GIANFORMAGGIO, «Legal Certainty, Coherence and Consensus: Variations of a Theme by MacCormick», en P. NERHOT (ed.), Law, Interpretation, 
la coherencia funciona como «un test de verdad o probabilidad en cuestiones de hecho y prueba acerca de las cuales no es posible obtener una prueba directa por medio de la observación inmediata» ${ }^{40}$. La sugerencia de MACCORMICK parece ser que un juez o jurado está justificado en aceptar la hipótesis acerca de los hechos que sea más coherente con las pruebas presentadas en el juicio y con un conjunto de principios explicativos ${ }^{41}$. Según este autor, es, en último término, el vínculo íntimo que existe entre la coherencia narrativa y la racionalidad lo que permite atribuirle a la primera fuerza justificativa en el contexto jurídico.

A diferencia de MACCORMICK, otros teóricos del derecho han defendido una concepción más amplia de la coherencia narrativa según la cual la coherencia narrativa es un estándar de justificación jurídica que es relevante tanto en relación a cuestiones fácticas como normativas. El modelo narrativo de la decisión judicial de B. JACKSON es un ejemplo prominente de este tipo de aproximación ${ }^{42}$. Las principales tesis del modelo propuesto por Jackson son las siguientes: i) la premisa mayor del silogismo judicial tiene forma narrativa; ii) la premisa menor se construye también como una narrativa, y iii) la relación entre la premisa mayor y la menor debe ser concebida como una relación de coherencia o de ajuste entre esquemas narrativos. Por tanto, según esta concepción de la decisión judicial, el proceso de toma de decisiones jurídicas consiste de manera principal en comparar la construcción narrativa de los hechos con el modelo narrativo que subyace a la norma jurídica aplicable ${ }^{43}$.

A pesar de que las propuestas brevemente examinadas en esta sección avanzan de manera significativa el proyecto de desarrollar una teoría coherentista de la prueba en el derecho, éstas no llegan a constituir una teoría completa de la coherencia. Además, estas teorías enfrentan, al igual que las teorías de la coherencia normativa, una serie de problemas que ponen en cuestión la viabilidad de las mismas. Paso ahora a describir los problemas principales que aquejan a las teorías de la coherencia en el derecho.

and Reality: Essays in Epistemology, Hermeneutics and Jurisprudence, Dordrecht, Kluwer, 1990, 402. Según GIANFORMAGGIO, es la noción de «consenso» en vez de la de «coherencia narrativa» la que justifica la reconstrucción de los hechos en el derecho.

40 MACCORMICK, «Coherence in Legal Justification», op. cit., 48. Según VAN RoERmund la concepción de la coherencia narrativa como un test de probabilidad, o plausibilidad, de los enunciados fácticos es demasiado restrictiva. Para este autor, «la coherencia narrativa» debe ser entendida como «el principio que estructura la relación entre hechos y normas (el caso y la decisión) no — como el profesor MACCORMICK parece sostenercomo el principio que ordena solamente uno de los términos de esta relación» (traducción mía). Vid. B. VAN Roermund, «On "Narrative Coherence” in Legal Contexts», en C. Faralli y E. Pattaro (eds.), Reason in Law, Milano, Giuffré, 1988. JACKSON también ha propuesto una teoría de la coherencia narrativa que no restringe el papel de la misma al ámbito fáctico. A diferencia de MACCORMICK, JACKSON sostiene que la teoría de la coherencia narrativa es aplicable tanto a la construcción de los hechos en el derecho como a la aplicación de las normas jurídicas. Vid. infra en esta sección.

${ }^{41}$ MACCORMICK ilustra cómo la coherencia narrativa funciona como un test para la justificación de las conclusiones fácticas en el contexto jurídico por medio del caso Rex vs. Smith. MACCORMICK, «Coherence in Legal Justification», op. cit., 49-50. Vid. también MACCORMICK, «The Coherence of a Case and the Reasonableness of Doubt», op. cit., 46-47; MACCORMICK, Legal Reasoning and Legal Theory, op. cit., 91-92.

42 Vid. JacKSOn, Law, Fact and Narrative Coberence, op. cit.; B. JACKSON, «Narrative Models in Legal Proof», International Journal for the Semiotics of Law, 1, 3, 1988; JACKSON, Making Sense in Law, op. cit.

43 JACKSON, Law, Fact, and Narrative Coherence, op. cit., 58-60. 


\section{ALGUNOS PROBLEMAS DE LAS TEORÍAS DE LA COHERENCIA EN EL DERECHO}

Las teorías de la coherencia en derecho son vulnerables a una serie de objeciones. Es interesante notar que estos problemas son comunes a las teorías coherentistas de la justificación de enunciados normativos y fácticos en el derecho, en otras palabras, los problemas del coherentismo en relación tanto con normas como con hechos tienen, como se verá, una estructura similar.

\subsection{Vaguedad}

El primer problema que enfrentan las teorías de la coherencia en el derecho es el problema de la vaguedad. La mayoría de las teorías de la coherencia no cuentan con un concepto bien definido de coherencia. Las teorías de la coherencia normativa están basadas en un concepto de coherencia demasiado vago para los propósitos de una teoría de la justificación jurídica ${ }^{44}$ y las teorías coherentistas del razonamiento jurídico acerca de los hechos apelan a una noción de coherencia narrativa que necesita especificarse de manera más detallada ${ }^{45}$.

\subsection{Holismo}

En segundo lugar, las teorías de la coherencia están comprometidas con un holismo radical que resulta bastante problemático. Según las teorías de la coherencia normativa, la justificación depende de la coherencia de «todo» el sistema de creencias acerca del derecho y de la moralidad política ${ }^{46}$. De manera similar, para las aproximaciones coherentistas al razonamiento acerca de los hechos en el derecho, «todo» el conjunto de creencias del decisor jurídico es relevante a la hora de determinar si una creencia acerca de los hechos está justificada ${ }^{47}$. Esta versión holista radical da lugar a tres problemas fundamentales. Primero, las teorías holistas son implausibles desde un punto de vista psicológico, ya que para llevar a cabo los cómputos globales de coherencia que exigen estas teorías es necesario tener unas capacidades cognitivas

${ }_{44}$ El esfuerzo más importante en la dirección de precisar y determinar los criterios de coherencia normativa ha sido llevado a cabo por Alexy y PeCZenik. Vid. AleXy y PeCZENIK, op. cit. Alexy y PeCZeniK han formulado una serie de criterios de coherencia que permiten evaluar la coherencia de un conjunto de proposiciones normativas. Sin embargo, esta teoría de la coherencia no da cuenta de cómo se pueden ponderar los distintos criterios cuando éstos entran en conflicto y, por ello, resulta, en un sentido importante, incompleta.

${ }^{4}$ Vid. TILlERS, «Web of Things in the Mind», op. cit., 1252; PARDO, op. cit., 401; TwINnING, Retbinking Evidence, op. cit., 241; y M. TARUfFo, La Prova dei Fatti Giuridici, Milano, Gifuffrè Editore, 1992, 285.

46 Vid. Dworkin, Law's Empire, op. cit., 253; R. Dworkin, «Natural Law Revisited», en J. ARTHuR y W. H. SHAw (eds.), Readings in the Philosophy of Law, New Jersey, Prentice Hall, 1993, 144; y A. PeCZENIK y J. C. HAGE, «Legal Knowledge of What?», Ratio Iuris, 13, 2004.

47 Las teorías coherentistas de la prueba están comprometidas con una versión del holismo según la cual las decisiones acerca de los hechos son fundamentalmente el resultado de una interacción entre la información presentada en el juicio y el conocimiento general del mundo del juez o miembro del jurado. Vid. J. JACKSON, «Analyzing the New Evidence Scholarship: Towards a New Conception of the Law of Evidence», Oxford Journal of Legal Studies, 16, 1996. 
y una memoria mucho mayor que la que tenemos los humanos ${ }^{48}$. Segundo, el holismo es descriptivamente inadecuado, puesto que los decisores jurídicos no ponen en juego todas sus creencias a la hora de resolver un determinado problema fáctico o normativo. Y, por último, este tipo de teorías son también insatisfactorias desde un punto de vista normativo, en cuanto que hacen depender la justificación de una decisión jurídica de la coherencia de un subconjunto de creencias morales ${ }^{49} \mathrm{y}$, en el caso del razonamiento acerca de hechos, parecen permitir que las decisiones se tomen en base a creencias que no están debidamente fundamentadas en las pruebas admitidas ${ }^{50}$.

\subsection{Circularidad y conservadurismo}

En tercer lugar, están los problemas relativos a la estructura de la justificación coherentista, en concreto, los problemas de la circularidad y del conservadurismo. Una objeción principal en contra de las teorías coherentistas de la justificación es que son viciosamente circulares. Es necesario mostrar que las teorías de la coherencia normativa no nos llevan a aceptar como justificado un determinado principio jurídico en virtud de su coherencia con un conjunto de materiales jurídicos cuya justificación, a su vez, depende de su coherencia con este mismo principio ${ }^{51}$. En el contexto del razonamiento jurídico acerca de hechos, es preciso mostrar que las teorías coherentistas no justifican una creencia en un enunciado probatorio en virtud de su coherencia con una determinada hipótesis que, al mismo tiempo, se considera como justificada en cuanto que es coherente con dicha prueba ${ }^{52}$.

Otro problema que enfrenta una concepción coherentista del razonamiento jurídico es el problema del conservadurismo. Las teorías de la justificación como coherencia son intrínsicamente conservadoras, ya que sostienen que la justificación de nuevos elementos depende de su coherencia con el conjunto de elementos previamente aceptado $^{53}$. Esta tendencia conservadora obstaculiza el cambio normativo y, en sistemas jurídicos deficientes desde un punto de vista moral, lleva a perpetuar la injusticia en aras de la coherencia ${ }^{54}$. En el ámbito fáctico, el conservadurismo inherente a las teorías de la coherencia puede conducir a interpretar las pruebas de manera que no perturben

48 Vid. B. LeVEnbOOK, «Coherence in Legal Reasoning», Law and Philosophy, 3, 1984, 371. KoRNBLITH ha argumentado en detalle que los modelos globales de coherencia son implausible desde un punto de vista psicológico, a la vista de las limitaciones cognitivas humanas. Vid. H. KoRnBLITH, «The Unattainability of Coherence», en J. BENDER (ed.), The Current State of the Coherence Theory: Critical Essays on the Epistemic Theories of Keith Lebrer and Laurence BonJour, Dordrecht, Kluwer,

49 Los modelos holistas de la coherencia se consideran generalmente como problemáticos en cuanto que hacen depender la justificación de la coherencia de todo el sistema de creencias, en vez de de la coherencia de un subconjunto relevante de creencias. Vid. A. Plantinga, Warrant: The Current Debate, Oxford, Oxford University Press, 1993, 112 y ss. 288-289.

50 Vid. W. Twining, The Great Juristic Bazaar, Dartmouth, Ashgate, 2002, 80; y TARUfFO, op. cit.,

51 Vid. L. MoraL, «A Modest Notion of Coherence in Legal Reasoning: A Model for the European Court of Justice», Ratio Iuris, 16, 2003, 319.

52 Vid., por ejemplo, WANEGAAR et al., Anchored Narratives, op. cit., 211.

53 Para una discusión excelente de este problema, vid. M. WiLliams, «Coherence, Justification, and Truth», Review of Metaphysics, 34, 1980.

54 Vid. J. RAZ, «Dworkin: A New Link in the Chain», California Law Review, 74, 1986. Vid. también R. WACKS, «Judges and Injustice», South African Law Journal, 101, 1984. 
la coherencia de las hipótesis que han sido previamente formuladas o, incluso, puede llevar a los decisores jurídicos a rechazar nuevas pruebas por razón de su incoherencia con las hipótesis aceptadas ${ }^{55}$.

\section{4. ¿Qué es la inferencia coherentista?}

Un cuarto problema que afecta a las teorías de la coherencia en el derecho es que las mismas no dan cuenta del tipo de inferencias que pueden usar los decisores jurídicos para construir una interpretación coherente de los materiales normativos relevantes o de las pruebas e hipótesis aducidas en el caso concreto. En otras palabras, las teorías de la coherencia en el derecho están incompletas, en cuanto que no especifican los mecanismos mediante los cuales los decisores jurídicos pueden llegar a construir la interpretación del derecho más coherente o la explicación de los hechos que mejor satisfaga los criterios de coherencia.

\section{5. ¿Por qué debe jugar la coherencia un papel en la justificación jurídica?}

Por último, una pregunta, sin duda, importante es la cuestión de por qué la coherencia debe jugar un papel en la justificación bien de los enunciados fácticos bien de los enunciados normativos en el derecho. Ésta es una pregunta de «segundo orden» que tiene que ver no con el problema de cómo funcionan los argumentos de coherencia sino con la cuestión de «por qué» la coherencia ha de tener fuerza justificativa en el contexto jurídico. A pesar de que la literatura acerca de la coherencia en el derecho se ha ocupado, en alguna medida, de este problema, las teorías de la coherencia no ofrecen una explicación detallada de las razones por las cuales la coherencia es un valor fundamental en el derecho.

¿Son estos problemas irresolubles? ¿Es entonces el coherentismo en el derecho demasiado problemático para ser considerado como una opción teórica plausible? De entrada, a la vista de estos problemas, ¿por qué empeñarse en desarrollar una teoría de la justificación como coherencia? Más adelante, ofreceré algunas razones por las cuales la coherencia es un valor importante en el derecho, pero quisiera esbozar aquí algunos argumentos a favor de la coherencia con el propósito de (espero) mostrar que, a pesar de sus problemas, el coherentismo es una aproximación a la justificación en el derecho que merece la pena desarrollar.

En primer lugar, incluso los más escépticos acerca del valor de la coherencia, están dispuestos a aceptar que la coherencia es, al menos, un ingrediente de la justificación. Se puede discutir el alcance de la misma como mecanismo de justificación, si juega un papel positivo o meramente negativo, si tiene la capacidad de generar justificación o si, por el contrario, sólo puede aumentar el grado de justificación, pero no conferirla. Sin embargo, que la coherencia es relevante para justificación es una tesis altamente

${ }^{5}$ Hay bastantes estudios empíricos que prueban que los decisores jurídicos, de hecho, utilizan estas estrategias para construir la coherencia cuando razonan acerca de los hechos en el derecho. Vid. D. SIMON, «A Psychological Model of Judicial Decision-Making», op. cit. 
plausible y generalmente aceptada. Si esto es así, entonces, incluso aunque no pudiera definirse la justificación exclusivamente en términos de coherencia, no parece que pueda analizarse de manera completa el problema de la justificación jurídica sin apelar a la idea de coherencia.

En segundo lugar, los argumentos de coherencia tienen un papel fundamental en los procesos de razonamiento y de decisión en diversos ámbitos; como dijo acertadamente ZIFF, «los humanos tenemos un gusto por la coherencia, somos expertos de la coherencia» ${ }^{56}$. Por ello, el coherentismo ha de resultar una opción atractiva para todo aquel que esté interesado en desarrollar teorías acerca de la argumentación jurídica que estén conectadas, de alguna manera, con nuestras capacidades naturales de razonamiento.

Por último, el coherentismo es una teoría alternativa a las aproximaciones formales al razonamiento jurídico — cuyos problemas y limitaciones han sido ampliamente discutidos en las últimas décadas- que no tiene las implicaciones escépticas de las tradicionales alternativas anti-formalistas, como el realismo jurídico y los movimientos críticos.

En conclusión, aunque los problemas del coherentismo son muchos y serios, hay buenas razones para explorar la posibilidad de desarrollar un modelo coherentista del razonamiento jurídico que tenga los recursos para resolver o, al menos, mitigar los problemas que afectan a las teorías coherentistas de la justificación jurídica. En la siguiente sección, trataré de desarrollar dicho modelo, tomando como punto de partida un estudio de las teorías de la coherencia que se han propuesto en diversas disciplinas y aplicando algunas de las ideas desarrolladas en estos campos al ámbito del derecho ${ }^{57}$.

\section{UN MODELO COHERENTISTA DE RAZONAMIENTO JURÍDICO}

El modelo coherentista de razonamiento jurídico que quisiera defender en este trabajo consta de cuatro elementos fundamentales: un concepto de coherencia en términos de satisfacción de restricciones, la idea de que la inferencia coherentista en el derecho es un tipo de inferencia a la mejor explicación, una concepción de la justificación jurídica como «coherencia óptima» y una aproximación contextualista a los estándares coherentistas de la justificación jurídica.

\subsection{La coherencia en el derecho como «satisfacción de restricciones»}

El primer ingrediente del modelo de coherencia que quisiera proponer aquí es una concepción de la coherencia para el derecho en términos de satisfacción de restricciones. P. THAGARD ha desarrollado una teoría de la coherencia como satisfacción de restricciones según la cual un conjunto de elementos es coherente cuando satisface

\footnotetext{
56 P. ZIFF, «Coherence», Linguistics and Philosophy, 7, 1984, 24.

57 Para una descripción detallada de las teorías de la coherencia defendidas en la filosofía y la lingüística, vid. AmaYA, The Tapestry of Reason, op. cit., capítulos 3 al 9.
} 
una serie de restricciones positivas y negativas ${ }^{58}$. THAGARD ha aplicado esta teoría general de la coherencia a una gran diversidad de campos, entre otros, la ética y la epistemología. Esta concepción general de la coherencia puede ser aplicada también con provecho, me parece, al ámbito del derecho. En el contexto jurídico, podríamos distinguir entre dos tipos de coherencia, la «coherencia normativa», es decir, el tipo de coherencia que es relevante para la justificación de los enunciados normativos y la «coherencia fáctica», i. e. el tipo de coherencia que es relevante para la justificación de los enunciados fácticos. Ambos tipos de coherencia pueden ser analizados tomando como base la teoría de THAGARD acerca de la «coherencia ética» y la «coherencia epistémica», respectivamente, aunque es necesario introducir algunas modificaciones para adaptar la teoría a las singularidades del contexto jurídico.

La coherencia fáctica requiere la interacción de los mismos tipos de coherencia que, según THAGARD, son relevantes para la justificación epistémica (a saber, la coherencia analógica, conceptual, perceptual, deductiva y explicativa) más un tipo de coherencia adicional, la coherencia deliberativa. Es necesario añadir este tipo de coherencia al conjunto de tipos de coherencia que son relevantes para la justificación de los juicios de hecho en el derecho porque, a diferencia de otros tipos de razonamiento acerca de hechos, el razonamiento acerca de los hechos en el derecho es, en último término, una parte de una instancia de razonamiento práctico acerca de si se debe o no condenar. Cada uno de estos tipos de coherencia se especifican, según THAGARD, a través de una serie de principios. Entre los distintos tipos de coherencia que son relevantes para la justificación de las conclusiones acerca de los hechos, la coherencia explicativa es, sin duda, el tipo de coherencia más importante. Según los principios de la coherencia explicativa, la coherencia explicativa es una relación simétrica, las restricciones positivas surgen de relaciones de analogía y explicación y las negativas, de relaciones de incompatibilidad y contradicción. Los elementos —en este caso, las proposiciones que describen pruebas e hipótesis acerca de los hechos- son aceptados si tal aceptación maximiza la coherencia, es decir, si maximiza la satisfacción de las restricciones impuestas por estos principios, teniendo en cuenta que — en virtud del llamado principio de prioridad- se le debe dar cierta preferencia a las proposiciones que describen pruebas. A estos principios habría que añadir algunos principios que yo llamaría «institucionales» con el fin de dar cuenta de la presunción de inocencia y de las restricciones impuestas por los estándares de la prueba.

La coherencia normativa resulta de la evaluación integrada de los mismos tipos de coherencia que, según THAGARD, son relevantes para la justificación moral (a saber, conceptual, perceptual, explicativa, analógica y deductiva) con una importante adición: la coherencia interpretativa. Es preciso añadir este tipo de coherencia porque el razonamiento jurídico es, de manera esencial, un tipo de razonamiento interpretativo. Este tipo de coherencia es, me parece, el tipo de coherencia que contribuye de manera más importante a la justificación de los enunciados normativos en el derecho. Los principios de la coherencia interpretativa son estructuralmente análogos a los principios de la coherencia explicativa, excepto que las restricciones positivas y negativas se establecen entre hipótesis interpretativas —en lugar de fác-

58 Thagard y K. Verbeurgt, «Coherence as Constraint Satisfaction», Cognitive Science, 22, 1998. 
ticas-y lo que podríamos llamar «elementos normativos» (precedentes, principios, etc.), que juegan un papel en el cálculo de la coherencia interpretativa similar al papel que juegan las proposiciones que describen pruebas en el cálculo de la coherencia explicativa.

El análisis de la coherencia en el derecho en términos de satisfacción de restricciones nos permite elaborar una concepción unitaria de las inferencias jurídicas, ya que la coherencia fáctica y la coherencia normativa comparten una misma estructura, a pesar de que los tipos de coherencia, los elementos y las restricciones relevantes sean diferentes. En base a este análisis, la inferencia jurídica, tanto acerca de proposiciones fácticas como normativas, podría describirse como un proceso de maximización de la coherencia que consta de los siguientes pasos o etapas: 1. La identificación de una «base» de coherencia, es decir, del conjunto de elementos que se pretende hacer coherente ${ }^{59}$. 2. La construcción de un conjunto de «contrastes» [contrast set] o, lo que es lo mismo, de un conjunto de alternativas de decisión plausibles ${ }^{60}$. 3. La elaboración de estas alternativas de decisión a través de una serie de mecanismos de construcción de la coherencia ${ }^{61}$. 4. La evaluación de las distintas decisiones posibles en virtud de los criterios de coherencia, normativa y fáctica, que se han propuesto anteriormente. 5. La selección como justificada de la decisión que mejor satisfaga los criterios de coherencia establecidos por las teorías de los tipos relevantes de coherencia, sobre todo, la coherencia explicativa, en el caso de los enunciados fácticos, y la coherencia interpretativa, en el caso de los enunciados normativos.

Este proceso de maximización de la coherencia se puede describir mejor como una «inferencia a la mejor explicación» mediante la cual los decisores jurídicos construyen un conjunto de hipótesis fácticas y normativas y seleccionan como justificada la hipótesis que es mejor, es decir, la hipótesis que es más coherente. La conceptualización de la inferencia coherentista como una inferencia explicativa es el segundo elemento del modelo coherentista del razonamiento jurídico que quisiera proponer, y que paso a analizar a continuación.

\subsection{Inferencia a la mejor explicación en el derecho}

El segundo elemento de esta propuesta coherentista es la tesis de que la coherencia se construye en el proceso de decisión jurídica a través de una «inferencia a la mejor explicación» ${ }^{62}$. La inferencia a la mejor explicación es un tipo de inferencia mediante

59 El término «base» de la coherencia es de RAz. Vid. RAZ, «The Relevance of Coherence», op. cit.

60 El término «contrast set» lo tomo prestado de JosEPHSON. Vid. J. JosEPHSON, «On the Proof Dynamics of Inference to the Best Explanation», en M. MACCRIMmON y P. TILLERS (eds.), The Dynamics of Judicial Proof, Heidelberg, Physica-Verlag, 2002, 293.

${ }^{61}$ Para un análisis detallado de estos mecanismos, vid. A. AMAYA, «Formal Models of Coherence and Legal Epistemology», Artificial Intelligence and Law, 15, 2007.

${ }_{62}$ Para una elaboración detallada de un modelo de inferencia a la mejor explicación en el derecho, vid. A. AmaYA, «Inference to the Best Explanation in Law», en H. KaPTEIN, H. PrakKEn y B. VerHeIJ (eds.), Legal Evidence and Proof: Statistics, Stories, Logic, Ashgate, Farnham, 2009. Acerca de la inferencia a la mejor explicación en el contexto de la prueba, vid. también K. ABimbOLA, «Abductive Reasoning in law: Taxonomy and Inference to the Best Explanation», The Dynamics of Judicial Proof, op. cit.; R. AlLEN y M. PARDO, «Juridical Proof and the Best Explanation», Law and Philosophy, 27, 2008. 
el cual se construyen y evalúan hipótesis explicativas ${ }^{63}$. Por tanto, la tesis que quisiera defender es que los decisores jurídicos forman creencias justificadas acerca de los hechos y las normas en el derecho a través de la construcción, en primer lugar, de una serie de hipótesis interpretativas y fácticas y, en segundo lugar, de la selección de una de ellas como justificada. Según esta concepción del razonamiento jurídico, la inferencia en el derecho es un proceso que consiste en dos etapas fundamentales, construcción y selección, que tiene carácter «eliminativo», es decir, que funciona por exclusión, y que es derrotable, ya que siempre es posible descubrir una explicación mejor que derrote a la explicación elegida.

La coherencia juega un papel fundamental tanto en la etapa de generación como en la de selección de hipótesis fácticas e interpretativas. En la etapa de construcción, la coherencia es una herramienta que ayuda a reducir el conjunto de hipótesis que se deben someter a consideración, de manera que las hipótesis que son altamente incoherentes con creencias empíricas y normativas básicas no se consideran lo suficientemente plausibles como para que merezca la pena evaluarlas posteriormente en la etapa de selección. En esta etapa, la coherencia también juega un papel primordial, ya que proporciona los estándares de evaluación necesarios para determinar cuál, entre las posibles alternativas, es la mejor hipótesis. La sugerencia, por tanto, es que la «mejor» explicación en el derecho es la explicación que mejor satisface los criterios de coherencia establecidos por las teorías de la coherencia fáctica y normativa.

Esta concepción explicativa de la inferencia jurídica, aunque - me parece- atractiva, no está exenta de problemas. A mi parecer, el problema principal que tiene que enfrentar un modelo de inferencia a la mejor explicación en el derecho es el llamado «problema de la consideración insuficiente» [problem of underconsideration] ${ }^{64}$. Según el modelo de la inferencia a la mejor explicación, el razonamiento jurídico funciona por exclusión y, como consecuencia de ello, la justificación jurídica depende, al menos en parte, de cuestiones relativas al contexto de descubrimiento. Pero el problema que surge entonces es el siguiente: ¿qué ocurre si el proceso de generación o descubrimiento de alternativas no es lo suficientemente bueno? En otras palabras, ¿qué excluye la posibilidad de que la mejor explicación sea simplemente la mejor alternativa de un conjunto deficiente de alternativas? El problema es que no parece que tengamos ninguna razón para aceptar la mejor hipótesis fáctica o normativa como justificada ya que es perfectamente posible que la hipótesis verdadera acerca de los hechos, o la mejor hipótesis interpretativa acerca del derecho, se encuentre entre aquellas alternativas que se han dejado de considerar. Este problema es, me parece, un problema que debe tomarse en serio. Lo que quisiera sugerir es que es posible solucionar el problema de la consideración insuficiente complementando el modelo de la inferencia a la mejor

63 Vid. G. HARMAN, «Inference to the Best Explanation», The Philosophical Review, 74, 1965; T. DAY y H. KinCAID, «Putting Inference to the Best Explanation in its Place», Synthese, 98, 1994; Y. BEN-MenAHEM, «Inference to the Best Explanation», Erkenntnis, 33, 1990; P. LIPTON, Inference to the Best Explanation, 2. ${ }^{\text {a }}$ ed., London y New York, Routledge, 2004; W. LYCAN, Judgment and Justification, New York, Cambridge University Press, 1988; y W. LYCAN, «Explanation and Epistemology», en P. K. Moser (ed.), The Oxford Handbook of Epistemology, Oxford, Oxford University Press, 2002.

${ }^{64}$ El problema de la consideración insuficiente ha sido formulado por B. VAN FraASSEN. Vid. B. C. VAN FraAsSEN, Laws and Symmetry, Oxford, Clarendon Press, 1989. P. LIPTON ha abordado este problema en «Is the Best Good Enough?», Proceedings of the Aristotelian Society, 43, 1993. Vid., tambien, S. Psillos, «On Van Frasssen's Critique of Abductive Reasoning», The Philosophical Quarterly, 46, 1996. 
explicación con una concepción responsibilista de la justificación ${ }^{65}$. En concreto, lo que quisiera argumentar es que un decisor jurídico está justificado en aceptar la mejor hipótesis fáctica o normativa —en contra de lo que sostiene la objeción - a condición de que haya construido el conjunto de hipótesis a considerar de un modo epistémicamente responsable. Me referiré a la hipótesis que un agente epistémicamente responsable identificaría como «mejor» como la hipótesis que es «óptimamente» coherente. Veamos ahora en qué consiste esta idea de «coherencia óptima» que, según el modelo propuesto, constituye el núcleo de la noción de justificación jurídica.

\subsection{La justificación jurídica como coherencia óptima}

El tercer elemento que me parece esencial en una teoría de la coherencia es la idea de que los juicios de responsabilidad epistémica son relevantes para la atribución de creencias justificadas acerca de los hechos y del derecho. En concreto, la tesis es que una hipótesis interpretativa o fáctica en el derecho es «óptimamente coherente» y, por tanto, está justificada si podría haber sido el resultado de un proceso epistémicamente responsable de maximización de la coherencia ${ }^{66}$. La responsabilidad epistémica requiere el cumplimiento de una serie de deberes epistémicos así como el ejercicio de un conjunto de virtudes epistémicas en el desempeño de las actividades de investigación y deliberación en el derecho ${ }^{67}$. Entre los deberes epistémicos que son relevantes para el razonamiento de tipo coherentista en el ámbito jurídico podríamos mencionar el deber de buscar más pruebas acerca de las proposiciones que son dudosas a la luz de las pruebas disponibles o el deber de creer sólo aquellas proposiciones que estén justificadas por las pruebas admitidas a juicio ${ }^{68}$. En el contexto de investigación acerca de los hechos y el derecho, para ser epistémicamente responsable es necesario el ejercicio de virtudes tales como la exhaustividad, la perseverancia en el seguimiento de una línea de investigación y la apertura de mente en la selección y evaluación de pruebas, mientras que en el contexto de deliberación acerca de los hechos y el derecho, algunas virtudes primordiales son la apertura a nuevas alternativas, la dis-

${ }^{65}$ Acerca de la distinción entre concepciones responsibilistas y no responsibilistas de la justificación, vid. J. PRYOR, «Highlights of Recent Epistemology», The British Journal for the Philosophy of Science, 52, 2001. Para una defensa de la concepción responsibilista de la justificación, vid. R. M. ChISHOLM, Theory of Knowledge, Englewood Cliffs, Prentice-Hall, 1977; y H. KorNBITH, «Justified Belief and Epistemically Responsible Action», The Philosophical Review, 92, 1983. Para una discusión contemporánea de las aproximaciones responsibilistas a la justificación, vid. M. STEUP (ed.), Knowledge, Truth and Duty: Essays in Epistemic Justification, Responsibility and Virtue, Oxford, Oxford University Press, 2001.

${ }^{66}$ He desarrollado en detalle esta propuesta en relación al ámbito fáctico en A. AmAYA, «Coherence, Justification, and Epistemic Responsibility in Legal Fact-finding», Episteme: A Journal of Social Epistemology, 5, 2008.

67 Acerca de los deberes epistémicos, vid. R. Feldman, «Epistemological Duties», The Oxford Handbook of Epistemology, op. cit. La literatura acerca de las virtudes epistémicas es muy amplia, vid., entre otros, L. ZAGZEBSKI, Virtues of the Mind: An Inquiry into the Nature of Virtue and the Ethical Foundations of Knowledge, Cambridge, Cambridge University Press, 1996; A. FaIRWEather y L. ZAGZEBSKI (eds.), Virtue Epistemology: Essays on Epistemic Virtue and Responsibility, Oxford, Oxford University Press, 2001; y G. AXTELL (ed.), Knowledge, Belief, and Character, Lanham, Rowman and Littlefield, 2000.

${ }^{68}$ Vid. R. J. Hall y C. R. Johnson, «The Epistemic Duty to Seek More Evidence», American Pbilosophical Quarterly, 35, 1998. La formulación clásica del deber de creer de acuerdo con las pruebas se debe a Clifford. Vid. W. K. Clifford, «The Ethics of Belief», The Ethics of Belief and Other Essays, New York, Prometheus Books, 1999. 
posición a considerar objeciones que pongan en cuestión las hipótesis preferidas y la habilidad de imaginar cómo las diferentes alternativas podrían afectar a las partes en el proceso ${ }^{69}$.

Ahora bien, a este punto, resulta pertinente preguntarse: ¿cuánta diligencia es necesaria para ser diligente en el contexto jurídico? ¿Qué pruebas es preciso procurar para cumplir con la obligación de buscar activamente razones para creer en una determinada hipótesis? ¿Qué nivel de cuidado es necesario para comportarse de una manera epistémicamente responsable? Una aproximación responsibilista a la justificación jurídica está incompleta a menos que exista alguna manera de determinar el nivel de escrutinio que es necesario satisfacer para poder tener creencias justificadas tanto acerca de los hechos como del derecho. La tesis que quisiera proponer aquí es que el contexto es esencial para determinar el nivel de cuidado debido y, por tanto, para fijar la severidad de los estándares de justificación jurídica. Este elemento contextualista es el cuarto - y último - de los componentes del modelo coherentista de razonamiento jurídico que pretendo articular y defender en este trabajo.

\subsection{Coherencia y contexto}

Los juicios de coherencia son altamente sensibles a rasgos contextuales, en otras palabras, cuando de coherencia se trata, como dice MARGOLIS, el contexto es la clave ${ }^{70}$. La idea fundamental que, me parece, es necesario incorporar en una teoría coherentista de la justificación jurídica es que los estándares de justificación varían con el contexto ${ }^{71}$. El rol del agente decisor, la importancia de lo que está en juego, las restricciones de carácter metodológico y dialéctico, los objetivos y los recursos disponibles son algunos de los rasgos contextuales que son especialmente relevantes a la hora de establecer la severidad de los estándares de justificación jurídica ${ }^{72}$. Por ejemplo, en la mayoría de los sistemas jurídicos, los estándares de justificación son más exigentes cuando el costo de tomar una decisión equivocada es muy alto, como es el caso en el derecho penal, particularmente, en relación con los delitos más graves. La tesis es, por tanto, que los mecanismos de tipo contextual juegan un papel fundamental en la determinación de la severidad de los estándares de justificación jurídica.

Los estándares coherentistas de la justificación jurídica se pueden hacer más o menos exigentes a través de la modificación de tres dimensiones centrales en una teoría de la coherencia: la base de la coherencia, la constitución del conjunto de contrastes y

69 Acerca de los rasgos de carácter que distinguen la conducta epistémicamente virtuosa, vid. N. COOPER, «The Intellectual Virtues», Philosophy, 69, 1994; J. MONTMARQUET, Epistemic Virtue and Doxastic Responsibility, Lanham, Rowman and Littlefield, 1993, 23-26; y ZAGZEBSKI, The Virtues of the Mind, op. cit., 114.

70 Vid. J. MARGOLIS, «The Locus of Coherence», Linguistics and Philosophy, 7, 1981.

71 Que los estándares de justificación están sujetos a variaciones que dependen del contexto es la tesis principal del contextualismo. Vid., entre otros, D. B. ANNIS, «A Contextualist Theory of Epistemic Justification», American Philosophical Quarterly, 15, 1978; S. CoHEN, «Knowledge and Context», The Journal of Philosophy, 83, 1986; D. LEWIS, «Elusive Knowledge», Australasian Journal of Philosophy, 74, 1996; y K. DeRose, «Contextualism: An Explanation and Defense», en J. GrECO y E. SOSA (eds.), The Blackwell Guide of Epistemology, Malden, Blackwell, 1999.

72 Para una discusión de los factores contextuales que son relevantes para la justificación, vid. M. WILLIAMs, Problems of Knowledge, Oxford, Oxford University Press, 2001. 
el grado de coherencia que se considera necesario para atribuir creencias justificadas ${ }^{73}$. Por ejemplo, el cargo y la condición de experto ayudan a determinar cuál es la base adecuada de la coherencia: mientras que en la elaboración de una teoría política o jurídica puede ser necesario hacer coherente un conjunto amplio de creencias acerca del derecho y de la moralidad política, en la toma de decisiones judiciales, la justificación de las mismas depende del grado de coherencia que tenga un conjunto de elementos mucho más reducido. De manera similar, el conjunto de alternativas que es preciso considerar para alcanzar creencias justificadas por virtud de su coherencia también depende del contexto. Por ejemplo, restricciones de carácter metodológico ayudan a configurar el conjunto de alternativas, destacando la importancia de algunas de éstas y determinando qué alternativas uno puede justificadamente ignorar. Para ilustrar el punto: mientras que la hipótesis según la cual es posible que la creencia de un testigo ocular sea producto de la alucinación es una hipótesis que debe ser seriamente considerada en el contexto de una investigación filosófica, esta hipótesis puede ser obviamente ignorada en un contexto jurídico. La severidad de los estándares de justificación también puede ser determinada incrementando o reduciendo el grado de coherencia que es necesario para la justificación. Por ejemplo, una determinada teoría del caso puede ser lo suficientemente coherente como para atribuir responsabilidad civil, pero ser, sin embargo, insuficientemente coherente para atribuir justificadamente responsabilidad penal.

En resumen, según el modelo propuesto, una hipótesis fáctica o interpretativa está justificada si es la más coherente de un conjunto de hipótesis alternativas que se ha construido de una manera epistémicamente responsable. Es posible alcanzar una solución «óptimamente coherente» a un determinado problema fáctico o normativo en el derecho a través de un proceso de inferencia a la mejor explicación. La coherencia es, según este modelo, una cuestión que depende de la satisfacción de una serie de restricciones y un estándar de justificación que varía con el contexto. Paso ahora a examinar cómo se podrían abordar, con los recursos de este modelo de coherencia, los problemas del coherentismo identificados anteriormente.

\section{LOS PROBLEMAS DEL COHERENTISMO: UNA PROPUESTA DE SOLUCIÓN}

La concepción de la coherencia en términos de satisfacción de restricciones nos permite solucionar el primer problema que se ha señalado antes, esto es, el problema de la vaguedad, ya que la concepción de la coherencia fáctica y normativa como satisfacción de restricciones nos proporciona una serie de criterios precisos para evaluar y comparar el grado de coherencia de las distintas alternativas.

73 El contexto no sólo impone un límite «mínimo» que debe ser satisfecho para poder tener creencias justificadas, sino también un límite «máximo», que asegura que el nivel de cuidado no se incremente a menos de que haya una razón especial para ello. Dado que hay limitaciones tanto de recursos cognitivos como de tiempo (límites que son particularmente visibles en el contexto jurídico), es importante evitar hacer trabajo extra, que no sea necesario en un determinado contexto de justificación. Como dice FogELIN, elevar los estándares de justificación conlleva una serie de «costes de transacción» que, como todos los costes, es preferible evitar. Vid. R. Fogelin, Walking the Tightrope of Reason, Oxford, Oxford University Press, 2003, 123-124. 
El problema de la circularidad sólo surge si uno acepta una concepción lineal de la inferencia según la cual la justificación es una propiedad que se transfiere de una creencia a otra a través de una cadena. Sin embargo, el modelo de coherencia propuesto rechaza esta concepción «tubular» de la justificación y se basa, por el contrario, en una concepción holista según la cual la justificación de una hipótesis fáctica o interpretativa depende de su coherencia con el resto de los elementos relevantes ${ }^{74}$. Además, en este modelo se asume que las relaciones de coherencia son simétricas y, por tanto, que dos elementos que son coherentes entre sí, son mutuamente interdependientes, y no que uno se infiere del otro. Por tanto, esta teoría evita el problema de la circularidad que es endémico a las teorías coherentistas, mostrando cómo las pruebas y las hipótesis fácticas, por un lado, y los elementos normativos y las hipótesis interpretativas, por el otro, se justifican mutuamente.

Este modelo también nos permite solventar el problema del conservadurismo. En el contexto del razonamiento acerca de los hechos, el modelo nos permite asegurarnos de que las pruebas juegan el papel que deben jugar en la selección de una hipótesis como justificada, ya que se da prioridad a las proposiciones que describen pruebas frente al resto de los elementos. Desde luego, siempre es posible rechazar estas proposiciones como injustificadas, si así lo requieren razones de coherencia. Sin embargo, esto es como debe ser: después de todo, ocasionalmente, tenemos buenas razones para dudar de la confiabilidad de las pruebas aducidas en un juicio. Sin embargo, el modelo garantiza que, a menos de que haya muy buenos argumentos de coherencia, los enunciados que describen pruebas sean aceptados y que, por tanto, éstos cumplan un papel fundamental en la determinación de la hipótesis acerca de los hechos que uno debe aceptar como justificada. En el contexto del razonamiento normativo, el modelo da prioridad a los enunciados que describen razones de autoridad y, en este sentido, sanciona una tendencia conservadora, al establecer que, para que una decisión esté justificada, ésta debe ser coherente con el conjunto de materiales autoritativos. Ahora bien, de hecho, que las razones de autoridad tengan un status privilegiado es un rasgo fundamental del razonamiento «jurídico» que diferencia a este tipo de razonamiento, precisamente, de otros tipos de razonamiento práctico. No puede ser, por tanto, un problema que la teoría de la coherencia reconozca el lugar prominente que tienen estas razones en la justificación de las proposiciones normativas. El problema surgiría, desde luego, si esta tendencia conservadora impidiera el cambio normativo, pero el modelo sí que da cabida al cambio normativo ya que permite rechazar las razones de autoridad siempre y cuando este rechazo aumente de manera significativa el grado de coherencia de las razones relevantes en su conjunto. Por tanto, este modelo exhibe un grado moderado de conservadurismo que es, me parece, una característica distintiva de nuestras prácticas de justificación jurídica.

La teoría de la coherencia propuesta aquí también nos permite solucionar los problemas derivados del holismo en cuanto que se fundamenta en una concepción contextualista de los estándares de justificación jurídica según la cual el conjunto de creencias que se tiene que hacer coherente no es todo el sistema de creencias del agente sino un subconjunto de las mismas que se considera relevante en el contexto particular. Esta

74 El término «tubular» [pipeline] es de SHogenjI. Vid. T. SHogENJI, «The Role of Coherence in Epistemic Justification», Australasian Journal of Philosophy, 79, 2001. 
aproximación contextualista a la justificación coherentista permite acercar este modelo a lo que los decisores judiciales pueden, de hecho, hacer, dadas nuestras limitaciones cognitivas; nos permite mejorar la adecuación descriptiva de la teoría, ya que explica de manera más adecuada los procesos de razonamiento que resultan en decisiones jurídicas justificadas; y al hacer que la justificación de una creencia acerca de los hechos o el derecho dependa exclusivamente de la coherencia de un subconjunto relevante de creencias solventa algunos de los problemas que surgen en relación a la adecuación normativa del coherentismo.

Otro problema que enfrentan las teorías de la coherencia es que las mismas carecen de una descripción detallada de la inferencia coherentista. El modelo propuesto soluciona este problema ya que proporciona una teoría acerca de los patrones inferenciales mediante los cuales los agentes jurídicos pueden llegar a tomar decisiones justificadas por virtud de su coherencia en términos de una inferencia a la mejor explicación. A pesar de que, sin duda, es necesario clarificar cómo operan las inferencias explicativas en el derecho, el modelo ofrece una descripción relativamente detallada de la manera en la cual funciona la inferencia coherentista en el derecho.

Por último, las teorías de la coherencia en el derecho no examinan de manera sistemática las razones por las cuales la coherencia es valiosa en el contexto jurídico. Como es bien sabido, una de las dificultades principales que enfrentan las teorías de la coherencia es la de demostrar que la coherencia nos conduce a la verdad (o a la corrección). A la luz de estas dificultades, pueden surgir dudas - legítimas, uno diría - acerca de si la coherencia tiene fuerza justificativa en el derecho. Para responder a la pregunta de por qué la coherencia confiere justificación en el derecho es necesario entrar a un debate de segundo orden acerca de cuáles serían las razones en base a las cuales se podrían justificar los estándares coherentistas de la justificación jurídica, es decir, es preciso ofrecer una meta-justificación adecuada de la concepción coherentista de la justificación jurídica. En la siguiente sección, ofrezco algunas razones por las cuales la coherencia es un valor importante en el razonamiento jurídico.

\section{EL VALOR DE LA COHERENCIA}

Para desarrollar un argumento a favor del valor de la coherencia en el razonamiento jurídico es necesario, en primer lugar, cuestionar una tesis acerca de qué es lo que hace que un determinado criterio de justificación sea valioso que está implícita en la mayor parte de las discusiones acerca de la justificación. Según esta tesis, un estándar de justificación es adecuado en la medida en que esté conectado de manera apropiada con la verdad y la corrección. Tomando esta tesis como punto de partida, se pone en tela de juicio que la coherencia pueda jugar un papel relevante en la justificación, dado que es dudoso que los estándares de coherencia puedan conducirnos a la verdad. Sin embargo, aunque, desde luego, uno de los criterios principales para evaluar un estándar de justificación es su capacidad para juzgar como justificadas creencias que son probablemente verdaderas, hay también otros criterios relevantes para determinar la adecuación de una teoría de la justificación. En lo que sigue, quisiera dar algunos argumentos a favor de una teoría coherentista de la justificación. 


\subsection{El argumento del anti-fundacionismo}

La primera razón a favor de una teoría coherentista de la justificación, como la que se ha propuesto aquí, es de carácter negativo. A la luz de las serias dificultades que presenta la principal alternativa al coherentismo, es decir, el fundacionalismo, no sólo en el ámbito jurídico sino también en los distintos campos en los que se han presentado propuestas fundacionistas (en epistemología, desde luego, pero también en ética, filosofía de la ciencia, etc.), la teoría de la justificación como coherencia parece, en principio, atractiva, aunque, desde luego, es necesario ofrecer algunas razones positivas a favor de esta teoría ${ }^{75}$.

\subsection{El argumento acerca de la conexión entre coherencia y verdad}

No parece que haya en las distintas propuestas coherentistas en diversos campos del conocimiento ningún argumento concluyente que demuestre que existe una conexión necesaria entre la coherencia y la verdad. Sin embargo, que no tengamos ningúna razón concluyente para creer que la coherencia conduce a la verdad, no significa que los esfuerzos por mostrar que la coherencia y la verdad están conectadas de manera adecuada sean inútiles. Por el contrario, hay un conjunto de estrategias diferentes que permiten concluir que la coherencia de nuestras creencias acerca del mundo no está desconectada de la verdad de las mismas ${ }^{76}$. Estas estrategias proporcionan, me parece, un buen punto de partida para construir un argumento que demuestre que aceptar conclusiones acerca de los hechos en el derecho en virtud de su coherencia es una buena manera de alcanzar conclusiones (probablemente) verdaderas. El problema de si la coherencia conduce a la verdad es mucho menos serio en el ámbito normativo que en el ámbito fáctico, dado que las teorías anti-realistas de la verdad de los enunciados normativos se

75 Para una crítica al fundacionismo acerca de la justificación epistémica, vid. W. ALsTON, Epistemic Justification, Ithaca, Cornell University Press, 1989; L. BonJour, The Structure of Empirical Knowledge, op. cit.; M. Williams, Groundless Belief, Princeton, Princeton University Press, 1999. Una clarificadora discusión de los problemas del fundacionalismo en el ámbito de la moral puede encontrarse en M. TIMMONS, «Foundationalism and the Structure of Ethical Justification», Ethics, 97, 1987.

${ }^{76}$ Los argumentos principales que tratan de conectar la coherencia y la verdad (en un sentido realista) son los siguientes. BONJOUR apela a una inferencia a la mejor explicación. Según BonJour, la mejor explicación de la coherencia y la estabilidad de un sistema de creencias que satisface el llamado «requisito de la observación» (que garantiza que un sistema de creencias atribuya una nivel alto de confiabilidad a una variedad razonable de creencias cognitivas espontáneas) es que el mismo corresponda (a largo plazo y de manera aproximada) al mundo exterior. LEHRER introduce un elemento externista en su teoría coherentista de la justificación que garantiza que una creencia justificada es también una creencia verdadera. Según LEHRER, para que una creencia esté justificada de manera no derrotable y, por tanto, para que una creencia constituya conocimiento, debe ser más razonable para un agente epistémico sostener esa creencia que rechazarla en base al llamado «ultra-sistema» de creencias, es decir, el conjunto de creencias verdaderas de dicho agente. THAGARD ofrece el siguiente argumento indirecto a favor de que la coherencia conduce a la verdad: las teorías científicas son, al menos, aproximadamente, verdaderas y los científicos usan argumentos de coherencia, por tanto, la coherencia conduce a la verdad. Por último, DAVIDSON sostiene que hay una presunción a favor de la verdad de una creencia que es coherente con el conjunto de creencias de un agente, ya que la mayoría de las creencias de un agente son verdaderas. Vid. BONJOUR, The Structure of Empirical Knowledge, op. cit., 169 y ss.; LEHRER, Theory of Knowledge, op. cit., 132 y ss; P. THAGARD, Coherence in Thought and Action, op. cit., 78 y ss.; y D. DAVIDSON, «A Coherence Theory of Truth and Knowledge», Subjective, Intersubjective, Objective, Oxford, Oxford University Press, 2001. 
consideran en general más plausibles que las teorías anti-realistas de la verdad de los enunciados fácticos y que los estándares coherentistas de la justificación se pueden combinar más fácilmente con teorías no realistas de la verdad que con teorías que defienden una concepción de la verdad como correspondencia. Por tanto, el problema de cómo se conectan la coherencia y la verdad no presenta, en principio, un obstáculo serio a las propuestas de analizar la justificación de las proposiciones normativas en el derecho en términos de coherencia. En conclusión, a pesar de que no se puede demostrar que una teoría de la coherencia nunca nos va a conducir por derroteros equivocados, ya que ciertamente nos puede llevar a aceptar creencias acerca de los hechos y el derecho falsas o incorrectas, hay buenas razones, dado el objetivo de alcanzar el valor de la verdad en el derecho, para aceptar la teoría de la justificación como coherencia.

\subsection{Coherencia y emoción}

Las emociones, tal y como han demostrado algunos estudios recientes, son un componente fundamental tanto en el razonamiento práctico como teórico en el derecho, al igual que en otras áreas ${ }^{77}$. Puesto que las relaciones de coherencia no tienen por qué ser relaciones entre elementos proposicionales y dado que los juicios de coherencia son sensibles a las respuestas emocionales, la teoría de la coherencia está mejor situada que otras teorías alternativas para dar cuenta del papel que juegan las emociones en la justificación jurídica ${ }^{78}$.

\subsection{El argumento de la plausibilidad psicológica}

Como han puesto de manifiesto algunos estudios empíricos, la teoría de la justificación como coherencia parece tener un alto grado de plausibilidad psicológica ${ }^{79}$. Ésta es una razón de peso a favor de esta teoría de la justificación. Desde una perspectiva naturalista, nuestras formas ordinarias de razonar constriñen el tipo de teorías normativas que son deseables; las teorías de la coherencia, a diferencia de otras teorías alternativas, satisfacen las restricciones impuestas por el naturalismo ${ }^{80}$. Además, uno de los objetivos, me parece, fundamentales, de una teoría del razonamiento jurídico

77 Vid. A. R. Damasio, Descartes' Error, New York, Putnam’s Sons, 1994.

78 THAGARD ha expandido su teoría de la coherencia para incorporar el papel que las emociones juegan en la toma de decisiones. Para una aplicación al derecho de esta teoría, P. THAGARD, «Why Wasn't O. J. Simpson Convicted?», Cognition and Emotion, 17, 2003.

${ }^{79}$ LIPTON ha interpretado los resultados experimentales que obtuvieron KAHNEMAN y TVERSKY en sus conocidos estudios sobre el razonamiento humano como prueba de la presencia de una fuerte tendencia al razonamiento de tipo explicativo. Vid. LIPTON, op. cit., 108-113. MoRAVSKI ha argumentado de manera persuasiva que la cognición puede ser vista como una actividad cuyo objetivo principal es el entendimiento y que, en un sentido importante, los humanos pueden ser caracterizados como bomo explanans. Vid. J. M. E. MORAVCSIK, Thought and Language, London y New York, Routledge, 1990. SimON, HolyOAK y sus colaboradores han mostrado que la toma de decisiones complejas se lleva a cabo mediante la construcción de relaciones de coherencia entre una serie de factores relevantes. Vid. K. J. HOLYOAK y D. SimON, «Bidirectional Reasoning in Decision-Making by Constraint Satisfaction», Journal of Experimental Psychology: General, 3, 1999; y DAN SIMON et al., «The Emergence of Coherence Over the Course of Decision-Making», Journal of Experimental Psychology: Learning, Memory, and Cognition, 27, 2001.

80 Para una clara introducción al naturalismo, vid. P. Godfrey-Smith, Theory and Reality, Chicago, Chicago University Press, 2003. Acerca de la historia del naturalismo, vid. P. KITCHER, «The Naturalists Return», 
es el de proporcionar una guía a los decisores jurídicos; la teoría de la coherencia, en cuanto que toma como punto de partida los procesos ordinarios de razonamiento, parece estar mejor situada para desempeñar esta función regulativa de los procesos de decisión jurídica que otras teorías que establecen patrones de razonamiento que están más alejados de las formas ordinarias que emplean los decisores jurídicos.

\subsection{Las dinámicas de la justificación}

Una ventaja de la teoría de la coherencia es que, a diferencia de otros modelos de justificación, esta teoría tiene los recursos necesarios para dar cuenta de los aspectos dinámicos de la justificación ${ }^{81}$.

\subsection{El valor práctico de la coherencia}

Las instituciones jurídicas están al servicio de una serie de objetivos y la coherencia es una herramienta valiosa para alcanzar algunos de ellos. Por ejemplo, la coherencia facilita la coordinación ${ }^{82}$, la efectividad ${ }^{83}$, la seguridad jurídica ${ }^{84}$, y la estabilidad social ${ }^{85}$ que son, sin duda, valores fundamentales en los ordenamientos jurídicos. Por tanto, parece haber razones importantes de orden práctico para promover el valor de la coherencia en el ámbito del derecho.

\subsection{El argumento acerca de la resolución del conflicto}

La coherencia está asociada a una concepción del razonamiento práctico según la cual es posible deliberar racionalmente acerca de los fines y no solamente acerca de cuáles son los mejores medios para lograr ciertos fines. Es decir, los métodos coherentistas no nos ayudan simplemente a realizar los distintos valores que se consideran centrales para el derecho, sino que son una herramienta fundamental para deliberar acerca de qué valores es importante perseguir y cómo sopesar los mismos cuando éstos entran en conflicto. Por tanto, la coherencia proporciona una guía inestimable para elegir entre distintas alternativas de decisión, tanto acerca de los hechos como del derecho, en casos en que hay conflicto de valores y, en este sentido, es de gran ayuda para el cumplimiento de la que es, quizá, una de las funciones más importantes del derecho, a saber, la resolución de conflictos a través de medios argumentativos ${ }^{86}$.

The Philosophical Review, 101, 1992. Una colección de artículos acerca del naturalismo puede encontrarse en H. KoRnBlith (ed.), Naturalizing Epistemology, Cambridge, MIT Press, 1993.

${ }_{81} \mathrm{~S}$. HAACK ha argumentado que el coherentismo es particularmente apropiado para dar cuenta de los aspectos dinámicos de la justificación en «A Foundherentist Theory of Empirical Justification», en L. POJMAN (ed.), The Theory of Knowledge, Belmont, Wadsworth, 1999.

82 Vid. M. E. Bratman, Intentions, Plans, and Practical Reasoning, Cambridge, Harvard University Press, 1987, 137. Vid. tambien RicHARDSON, op. cit., 152-158.

83 Vid. Thagard y Millgram, «Deliberative Coherence», op. cit., 67.

${ }^{84}$ Vid. Moral, «A Modest Notion of Coherence in Legal Reasoning», op. cit., 320.

85 Vid. Alexy y PeCZENIK, op. cit., 145.

86 Dice ATIENZA: «El derecho puede verse por ello (aunque ésta no sea la única perspectiva posible) como una compleja institución volcada hacia la resolución (o el tratamiento) de conflictos por medios argumentativos 


\subsection{El valor constitutivo de la coherencia}

La coherencia es constitutiva de la identidad individual y política ${ }^{87}$. Los decisores jurídicos, por tanto, no pueden sin más desatender las exigencias de la coherencia porque si así lo hicieran estarían, de hecho, negándose a determinar su propia identidad como miembros de una comunidad política. Esta dimensión constitutiva de la coherencia es una razón fundacional para valorar la coherencia como un estándar que debe guiar a los decisores jurídicos en el desempeño de sus funciones.

Estos argumentos, considerados en su conjunto, dan un apoyo importante a la teoría de la justificación como coherencia. Dado que parece haber buenas razones para la coherencia y puesto que, como he argumentado anteriormente, los problemas del coherentismo no son de imposible resolución, una teoría de la justificación que le dé un papel importante a la coherencia, como la que se ha propuesto en este trabajo, parece ser un buen candidato para una teoría de la justificación jurídica. Después de haber articulado y defendido un modelo coherentista del razonamiento jurídico, pasemos ahora a examinar algunas de las implicaciones que tiene este modelo para la teoría general de la argumentación jurídica.

\section{COHERENCIA, RAZONAMIENTO JURÍDICO Y TEORÍA DEL DERECHO}

El modelo de coherencia propuesto tiene, me parece, algunas implicaciones interesantes para la teoría general de la argumentación jurídica y, de manera más amplia, para la teoría del derecho. Esta propuesta coherentista está basada en una concepción de la justificación jurídica que se aparta, en algunos aspectos centrales, de la concepción de la justificación que caracteriza a la teoría estándar de la argumentación jurídica.

En primer lugar, el modelo de coherencia articulado y defendido en las secciones anteriores, se basa en una concepción anti-fundacionista de la justificación jurídica, es decir, este modelo niega que exista un conjunto privilegiado bien de proposiciones fácticas bien de proposiciones normativas que confieran justificación al resto de proposiciones acerca de los hechos o el derecho. En este sentido, este modelo se aparta tanto de las concepciones positivistas formales del razonamiento jurídico como de las aproximaciones probabilísticas al razonamiento acerca de los hechos en el derecho, que están basadas en una epistemología fundacionista. El modelo propuesto también se aleja de otras concepciones anti-fundacionista que se han defendido en la literatura jurídica — como la de DwORKIN- en cuanto que, a diferencia de éstas, el modelo otorga - a través del principio de prioridad - cierta preferencia a las proposiciones que describen razones de autoridad así como a los enunciados probatorios, aunque, en último término, su justificación depende, al igual que la del resto de las proposiciones, de las relaciones de coherencia que éstas tengan con los otros elementos fácticos y nor-

y en las distintas instancias de la vida jurídica». Vid. M. ATIENZA, El derecho como argumentación, Barcelona, Ariel, 2006, 59.

${ }_{87}$ HuRLEy ha defendido en detalle que la coherencia tiene un valor constitutivo de la identidad personal y comunitaria en su libro Natural Reasons, op. cit., especialmente, en el capítulo 13. 
mativos. De este modo, frente a otros modelos coherentistas, que conciben la justificación jurídica como un problema «puro» de coherencia, el modelo propuesto entiende la justificación jurídica como un problema «discriminatorio», reconociendo el papel fundamental que deben jugar las razones de autoridad y las pruebas en la justificación jurídica de las proposiciones normativas y fácticas, respectivamente ${ }^{88}$.

En segundo lugar, el modelo de coherencia propuesto está basado en una concepción contextualista de la justificación jurídica según la cual los estándares de justificación jurídica de las conclusiones acerca de cuestiones tanto de hecho como de derecho dependen de factores contextuales, tales como los objetivos relevantes, la importancia del caso, las consecuencias de la decisión o las restricciones de tiempo y recursos. En este sentido, este modelo se aleja de la concepción de la justificación dominante en teoría del derecho según la cual es posible definir los estándares de justificación jurídica de manera general, sin atender a los rasgos específicos del caso concreto, y contribuye al desarrollo de aquellas aproximaciones, todavía minoritarias, que tratan de articular estándares de justificación jurídica sensibles al contexto.

En tercer lugar, esta propuesta coherentista está fundamentada en una concepción responsibilista de la justificación jurídica, según la cual, los estándares de justificación jurídica no son independientes de los estándares de responsabilidad que regulan la deliberación de los decisores jurídicos. En este punto, el modelo propuesto se aleja de manera fundamental de las teorías estándar de la justificación jurídica, en las que el estudio de los deberes y virtudes de los decisores jurídicos se considera una materia que cae, de manera característica, fuera de su ámbito de aplicación. Por el contrario, según la propuesta defendida aquí, el análisis de los estándares de responsabilidad es una parte fundamental de la teoría de la justificación jurídica. El modelo propuesto, por tanto, permite apreciar que existen importantes conexiones entre la teoría de la argumentación jurídica y la ética profesional. En cuanto que el modelo de coherencia propuesto defiende una concepción de los estándares de responsabilidad en la que juegan un papel importante tanto los deberes como las virtudes, este modelo destaca la relevancia de nociones aretaicas para una teoría de la justificación jurídica y, de manera más general, apunta la posibilidad de desarrollar una jurisprudencia de la virtud ${ }^{89}$, como una alternativa aún por explorar a las tradicionales teorías del derecho de corte kantiano o consecuencialista.

En cuarto lugar, según el modelo coherentista propuesto, el razonamiento jurídico - tanto acerca de normas como de hechos- es un razonamiento primordialmente de tipo explicativo. Aunque el razonamiento explicativo se ha discutido en alguna medida en la literatura jurídica, la discusión se ha centrado en el papel que la abducción juega en el descubrimiento de hipótesis fácticas ${ }^{90}$. Este modelo, sin embargo, se basa en

${ }^{88}$ Acerca de la distinción entre problemas puros de coherencia y problemas discriminatorios de coherencia, vid. THAGARD, Coherence in Thought and Action, op. cit., 70-72.

${ }^{89}$ El término «virtue jurisprudence» ha sido acuñado por Solum. Vid. L. Solum, «Virtue Jurisprudence: A Virtue-Centred Theory of Judging», en M. BRADy y D. PRITChard (eds.), Moral and Epistemic Virtues, Malden, Blackwell, 2003. Para una colección de ensayos acerca de la jurisprudencia de la virtud, vid. Virtue Jurisprudence, L. Solum y C. FarRelly (eds.), New York, Palgrave MacMillan; y Virtue, Law, and Justice, A. AmaYa y H. Ho (eds.), Oxford, Hart Publishing, 2012.

${ }_{90}$ Vid. D. A. Schum, «Species of Abductive Reasoning in Fact Investigation in Law», The Dynamics of Judicial Proof, op. cit.; y D. SCHUM, «Marshalling Thoughts and Evidence during Fact-Investigation», South Texas Law Review, 40, 1999. 
una concepción más amplia de la inferencia explicativa según la cual la inferencia a la mejor explicación es un tipo de inferencia que juega un papel importante no sólo en el contexto de descubrimiento sino también en el de justificación y que, además de tener una importancia central en el razonamiento acerca de los hechos en el derecho, es un patrón de inferencia fundamental en el razonamiento normativo. En este sentido, este modelo de coherencia saca a la luz los importantes paralelismos estructurales que parecen existir entre las inferencias fácticas y normativas en el derecho y, por tanto, apunta a la posibilidad de desarrollar una teoría unitaria para ambos tipos de inferencia.

En quinto lugar, este modelo se basa en una concepción naturalista de la justificación jurídica, es decir, en una determinada concepción acerca de la relación entre las cuestiones normativas y las descriptivas según la cual para determinar cómo deben razonar los decisores jurídicos es indispensable tomar en consideración las investigaciones de tipo empírico acerca de cómo, de hecho, se toman las decisiones jurídicas. En este sentido, este modelo cuestiona la tesis - generalmente asumida en las teorías estándar de la argumentación jurídica - según la cual hay una separación fundamental entre la psicología de los procesos de decisión jurídica y las cuestiones acerca de la justificación de las decisiones jurídicas e invita a considerar el posible impacto de los recientes desarrollos en psicología del razonamiento en la elaboración de una teoría normativa de la argumentación jurídica ${ }^{91}$.

Por último, el modelo coherentista propuesto se basa en una concepción amplia de la racionalidad práctica según la cual el razonamiento jurídico no es un razonamiento exclusivamente instrumental sino que, por el contrario, es también un razonamiento acerca de fines y valores. De este modo, este modelo se aparta de las aproximaciones tanto formalistas como realistas según las cuales el razonamiento jurídico es un razonamiento de tipo medio-fin y se inscribe en el conjunto de teorías que defienden que la deliberación en contextos jurídicos consiste, al menos en parte, en razonar acerca de fines y valores. Este modelo, en cuanto que presenta la inferencia coherentista como una forma no instrumental de razonamiento práctico, pretende, por tanto, contribuir, en alguna medida, al estudio de los patrones argumentativos mediante los cuales se razona acerca de fines y valores en el derecho.

\section{UN CATÁLOGO DE PROBLEMAS ABIERTOS}

La teoría coherentista a la justificación jurídica presentada en este trabajo tiene, me parece, algunos rasgos deseables. En primer lugar, esta teoría tiene un ámbito de aplicación más amplio que algunas de las aproximaciones coherentistas al razonamiento jurídico, ya que es, en principio, aplicable tanto al razonamiento acerca de cuestiones de hecho como de cuestiones de derecho. Además, esta teoría parece plausible desde un punto de vista psicológico, puesto que está basada en una concepción de la coherencia como satisfacción de restricciones y, como han mostrado algunos estudios recientes

${ }_{91}$ Ha habido, no obstante, algún intento aislado por desarrollar el programa naturalista en el derecho, vid. B. LeITER, «Naturalism and Naturalized Jurisprudence», en B. BIX (ed.), Analyzing Law: New Essays in Legal Theory, Oxford, Clarendon Press, 1998. En el contexto del derecho de la prueba, vid. AlLEN y LeiTer, op. cit. 
en psicología cognitiva, una gran parte de los procesos cognitivos humanos puede ser entendida en términos de redes conexionistas, como las que se usan para representar los mecanismos de satisfacción de restricciones. Por último, aunque - huelga decirlo- el modelo propuesto no soluciona de una manera plenamente satisfactoria los problemas tradicionales del coherentismo, me parece que, al menos, mitiga de manera significativa la importancia de los mismos. De todos modos, el modelo, desde luego, no está exento de problemas. Para concluir, quisiera señalar someramente algunas de las dificultades que tiene esta propuesta coherentista.

En primer lugar, el análisis de la coherencia en términos de satisfacción de restricciones tiene algunos problemas que no son, me parece, menores, tales como el problema de cómo generar el input para el cálculo de coherencia o el problema de cómo integrar los distintos tipos de coherencia que son relevantes para la justificación jurídica.

En segundo lugar, el análisis de las inferencias explicativas en el derecho presentado en este trabajo apenas toca algunos de los aspectos generales de los mecanismos mediante los cuales es posible construir la coherencia en la toma de decisiones jurídicas. Es necesario analizar en mucho más detalle los procesos de descubrimiento de hipótesis interpretativas y fácticas novedosas así como el problema de cómo las inferencias a la mejor explicación confieren justificación a sus conclusiones en contextos jurídicos, por nombrar sólo algunas de las cuestiones que tendrían que ser atendidas.

En tercer lugar, en este trabajo se ha defendido una concepción responsibilista de la justificación jurídica según la cual los estándares de responsabilidad epistémica son relevantes para la atribución de creencias justificadas en el derecho. Sin embargo, la articulación en detalle de esta concepción de la justificación jurídica presenta muchos problemas. Entre otros, es esencial investigar el problema de si los conceptos deónticos y aretaicos en base a los cuales se han definido los estándares de responsabilidad epistémica conectan de manera adecuada con la verdad y la corrección de los enunciados fácticos y normativos en el derecho, respectivamente.

En cuarto lugar, una versión contextualista de la teoría coherentista de la justificación jurídica, como la que se ha defendido aquí, abre un flanco al relativismo. Para poder liberar a la teoría de la coherencia de sospechas escépticas o relativistas, es necesario especificar en detalle los rasgos del contexto que son relevantes para los juicios de coherencia así como aclarar cómo se individualizan los contextos en el derecho.

En quinto lugar, una concepción naturalizada de la justificación jurídica puede parecer, en principio, incompatible con la normatividad de la misma. Es preciso, por tanto, mostrar que el programa naturalista, entendido correctamente, no implica desproveer de normatividad a la teoría de la justificación jurídica.

Por último, se podría objetar que el modelo de justificación propuesto aquí asume una concepción excesivamente intelectualizada de los procesos de decisión jurídica, en cuanto que otorga un papel fundamental a las virtudes epistémicas y a las habilidades intelectuales de los decisores jurídicos. Es necesario, por tanto, clarificar cómo las habilidades epistémicas y morales contribuyen a la corrección de las decisiones jurídicas y, en concreto, cómo los deberes epistémicos y morales, por un lado, y las virtudes intelectuales y morales, por otro, se relacionan entre sí. 
Éstos son algunos de los problemas que sería necesario abordar para poder desarrollar una concepción coherentista de la justificación jurídica basada en los principios generales expuestos en este trabajo. Estos problemas están ligados a cuestiones más amplias, tales como el posible impacto de los estudios en ciencias cognitivas en la teoría del derecho, la naturaleza derrotable del razonamiento explicativo, la relación entre contextualismo y relativismo en el derecho, las implicaciones del naturalismo para la normatividad de la teoría del derecho, o la relevancia de la teoría de la virtud para la teoría de la justificación jurídica. En este sentido, espero que, al menos, el modelo propuesto sugiera algunas líneas de investigación interesantes tanto en la teoría de la coherencia como, de manera más general, en el ámbito de la teoría del derecho. 